\title{
La Política de Cohesión Europea en dos regiones españolas: Configurando las redes de políticas y el capital social
}

\author{
Jacint Jordana \\ Fabiola Mota \\ Andrea Noferini
}

Resumen: El artículo analiza la fase de programación de la política europea de cohesión centrándose en el nivel autonómico de gobierno. Sostiene que los Fondos Estructurales han afectado de manera esencial la configuración de las políticas de desarroIlo regional. En particular, la europeización de la política regional en España ha implicado un papel creciente de los gobiernos autonómicos, que han ido adoptando un conjunto de prácticas y procedimientos homogéneos en los procesos de programación e implementación de la política de cohesión. No obstante, la evidencia empírica recogida en las comunidades autónomas de Galicia y de Murcia, en relación con la programación del período financiero 2007-2013 (PORs), prueba la existencia de diferentes configuraciones de la red de política regional que llevan a cabo las tareas de programación. Al mismo tiempo, también las características de capital social de los actores implicados en cada red de política regional presentan diferencias significativas. Estos resultados sugieren que, a pesar de que los procedimientos formales de programación son prácticamente idénticos, los procesos políticos son bastante distintos en cada comunidad autónoma, lo que plantea nuevos interrogantes sobre las causas de dichas variaciones.

Palabras clave: Políticas de cohesión, policy networks, capital social, comunidades autónomas, fondos estructurales, desarrollo regional, gobernanza europea multinivel.

* Este artículo ha sido posible gracias al apoyo del proyecto SOcCOH, The Challenge of Socioeconomic Cohesión in the Enlarged European Union, financiado por el VI Programa Marco de Investigación de la Unión Europea (Ref.029003). Agradecemos a todas las personas entrevistadas su disponibilidad, y.en particular a Juan Josè Lirón Lago y a Xoan Vázquez Mao por su apoyo y cordialidad. También agradecemos a la Xunta de Galicia y al Gobierno de la Región de Murcia el interés mostrado en nuestra investigación. Los errores y omisiones son responsabilidad de los autores. 


\section{LA POLÍTICA REGIONAL EN ESPAÑA: UN ESTUDIO COMPARADO}

\subsection{Introducción}

Desde su ingreso en la Unión Europea en 1986, la política regional española se ha ido ajustando a la lógica multinivel que caracteriza al proceso europeo de elaboración de políticas públicas. Observamos múltiples niveles de gobierno involucrados en la definición e implementación de las políticas de desarrollo regional, conformando una estructura política compleja que incorpora tanto a actores públicos como no públicos, y que comporta la distribución de importantes cantidades de recursos económicos hacia las regiones menos desarrolladas. En dicho proceso se han generado espacios significativos para que los gobiernos nacionales y sub-nacionales definieran estructuras concretas de elaboración de políticas y procedimientos de planificación.

En este artículo abordamos la elaboración de las políticas regionales basadas en los fondos estructurales europeos, prestando especial atención al proceso de definición y programación correspondiente a los Programas Operativos Regionales (PORs) para el periodo 2007-2013. En particular, estudiamos el papel desempeñado por los diversos actores en su elaboración y las dinámicas efectivas de la participación, más allá de los comités y consejos establecidos formalmente. Nuestro principal objetivo es analizar las redes de actores involucrados en este proceso identificando, por una parte, los vínculos entre los actores relevantes y sus percepciones mutuas; y por otra, las características de los actores relativas al capital social, para discutir como pueden afectar al proceso general de formulación de las políticas.

La investigación empírica se ha realizado en dos comunidades españolas: Galicia y Murcia. Ambas regiones presentan un nivel de desarrollo socioeconómico muy similar (por ejemplo, en términos de su PIB per cápita y del nivel de capital social de la población). Estas semejanzas nos permiten controlar a priori la influencia de este tipo de factores en la emergencia de distintos modelos de elaboración de políticas de desarrollo regional en España, y concentrarnos en las pautas de interacción entre los actores implicados. A pesar de la homogeneidad inherente a los procedimientos de la Política de Cohesión europea, nuestra investigación constata la existencia de modelos distintos de elaboración de políticas regionales a los cuales corresponden también diferentes estructuras de policy networks.

Los datos utilizados en la investigación proceden de fuentes distintas. En primer lugar, hemos analizados los principales documentos oficiales de los diversos actores institucionales (en sus distintos niveles de gobierno), como el Marco Comunitario de Apoyo (2000-2006), el Marco Estratégico Nacional de Referencia (2007-2013), las Estrategias de Desarrollo Regional, los Programas Operativos o las Evaluaciones Intermedias de los programas. En segundo lugar, la información ha sido obtenida mediante entrevistas en profundidad y cuestionarios estructurados realizados a los principales actores regionales (funcionarios públicos, miembros de los Comités de 88 Seguimiento de los PORs, representantes de los gobiernos locales, asociaciones em- 
presariales, sindicatos, ONGs, etc.). Estas entrevistas nos proporcionaron la información para el análisis de redes y de las características de capital social de los actores involucrados en las mismas. Los entrevistados fueron identificados de acuerdo con dos criterios: su posición y su reputación en la red de políticas públicas. Puesto que centramos el interés en el proceso de políticas regionales a nivel de comunidad autónoma, descartamos incluir a los actores nacionales en el análisis de redes. Comenzamos la identificación de los actores seleccionando a aquéllos que ocupaban un cargo oficial en el proceso de planificación de la Política de Cohesión 2007-2013 y a aquéllos que, en entrevistas previas a expertos, habían sido identificados como actores relevantes en determinadas áreas de políticas en cada región. Un segundo paso nos llevó a identificar aquellos actores que por su reputación, aunque no tuviesen responsabilidades oficiales, habían tenido un papel relevante en alguna de las fases del proceso de definición de la política. Se prestó también particular atención a las relaciones entre distintas unidades administrativas dentro de la región, y también a las relaciones entre éstas y terceros.

\subsection{Selección de las regiones: Galicia y Región de Murcia}

Las comunidades de Galicia y de Murcia comparten una ubicación periférica en el territorio español además de trayectorias históricas de relativo subdesarrollo económico en comparación con la media regional española y, por supuesto, europea. No obstante, y gracias a los patrones de crecimiento económico sostenidos experimentados por la economía española durante las últimas dos décadas, Galicia y Murcia han convergido de manera parcial hacia el promedio regional europeo. En 1986 ambas regiones presentaron niveles de PIB per cápita cercanos al 60\% del promedio de los 15 miembros de la UE. Actualmente, Galicia y Murcia han alcanzado respectivamente, el $84,2 \%$ y el $87,7 \%$ de los niveles de PIB per cápita de los 27 miembros de la UE (Eurostat 2008).

Durante las dos últimas décadas, Galicia y Murcia han experimentado un profundo proceso de reestructuración económica que ha abierto las puertas a la modernización (Alcaide y Alcaide 2003; Serrano 2005). Ha sido especialmente notable la transformación de sus estructuras económicas tradicionales -fuertemente dependiente del sector agrícola- en economías más modernas e industrializadas. Sin embargo, los patrones de crecimiento han seguido diversos recorridos. Murcia, en particular, ha mostrado un rendimiento extraordinariamente alto con tasas de crecimiento anual superiores al promedio español para todo el período 1995-2004. En cambio, Galicia ha crecido de manera más moderada y siempre por debajo de los parámetros nacionales (Figura 1). No obstante, y como resultado del proceso de industrialización, en ambas regiones ha disminuido de manera importante el peso relativo del sector primario mientras que el sector de servicios ha crecido de manera impresionante. De las dos comunidades, Murcia se encuentra más claramente orientada hacia las exportaciones, contando para ello con una potente industria agro-alimentaria (sector que ocupa al 31\% de la fuerza de trabajo industrial) que contribuye en una alta proporción a la riqueza regional (Región de Murcia 2004). 
FIGURA 1

Convergencia regional, 1985-2005 (UE-15=100)

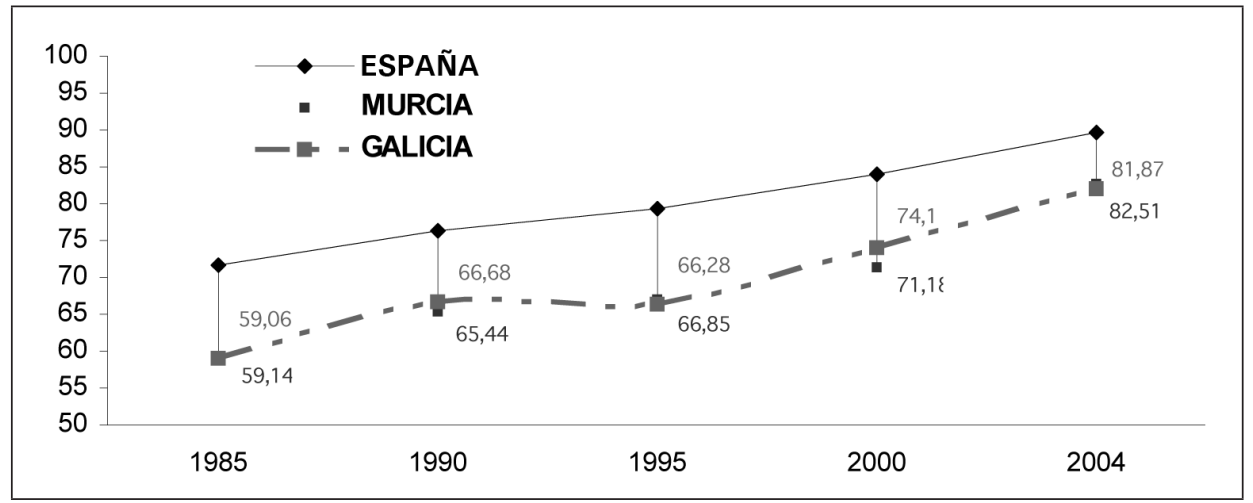

Fuente: Elaboración propia a partir de Piedrafita, Steinberg y Torreblanca (2007).

En el plano político, las dos comunidades han mostrado un grado de estabilidad política similar, contando con gobiernos regionales duraderos que generalmente han repetido más de un mandato. Desde las primeras elecciones regionales, en 1981, la Xunta de Galicia ha estado gobernada durante más de veintiun años por el principal partido conservador español (primero UCD y luego PP). En el 2005, tras catorce años de gobierno ininterrumpido del Partido Popular, llegó al poder una nueva coalición mayoritaria formada por el PSdG-PSOE y el BNG. En Murcia, el escenario político es aún más estable. Desde las primeras elecciones regionales, en 1983, Murcia ha celebrado siete elecciones autonómicas. La primera parte de este período estuvo dominada por sucesivos gobiernos formados por el PSOE y a partir de 1995 por gobiernos del PP. En general, la política regional en Murcia muestra una evolución acorde con la política nacional española (la ausencia de partidos políticos de ámbito exclusivamente regional ha favorecido esta coincidencia), si bien las últimas elecciones regionales de 2007 reforzaron la posición de gobierno del Partido Popular a pesar del la presencia del PSOE en el gobierno central.

\subsection{La Política de Cohesión en Galicia y la Región de Murcia}

Cuando España ingresó en la CE, tanto Galicia como Murcia eran elegibles para recibir fondos estructurales como regiones de Objetivo 1. Desde entonces, ambas regiones han experimentado, y ya han completado, tres períodos financieros (19891993; 1994-1999; 2000-2006). Bajo la nueva regulación para el período 2007-2013, Murcia superó (estadísticamente) el umbral del 75\% de PBI per cápita y es una región con una reducción progresiva dentro del Objetivo de Convergencia (Phasing-Out). 90 tivo. 
En Murcia, la Política de Cohesión es generalmente contemplada como uno de los principales factores explicativos del crecimiento económico. Durante el período 1989-2006, y estando clasificada como una de las regiones menos favorecida, la Región de Murcia recibió alrededor de 3.000 millones de euros a través de los fondos estructurales. Los flujos financieros europeos fueron destinados básicamente a dos objetivos: infraestructura de transporte y modernización del sector agrícola. Respecto al primero, si antes del ingreso en la UE Murcia no disponía de ninguna vía de comunicación importante, en la actualidad 500 kilometros de autovías atraviesan su territorio gracias al dinero proveniente desde Brusela. La principal consecuencia ha sido un notable incremento de la movilidad intra e inter regional. El segundo objetivo perseguía la transformación del sector agrícola tradicional y el diseño de un nuevo sector agrícola moderno. La liberalización económica y las presiones sobre la competitividad llevaron a las elites económicas regionales a invertir en sectores orientados a la exportación. Es importante subrayar que la asistencia financiera europea en la Región de Murcia contribuyó también a mitigar el costo social de la reestructuración industrial: los fondos estructurales sirvieron para mantener y/o crear 5.000 puestos de trabajo nuevos cada año en el mercado laboral (Región de Murcia, 2004). La ampliación de la UE ha afectado profundamente a la Región, especialmente teniendo en cuenta que para el período financiero 2007-2013 recibirá cerca de 826 millones de euros, con una reducción de aproximadamente el 35\% de la ayuda estructural'.

Los fondos estructurales han representado un factor clave de desarrollo también en Galicia. La clasificación de Galicia como región de Objetivo 1 desde 1986 significó un aumento en la suma de fondos disponibles para programas de desarrollo. La cantidad total asignada en el período 1989-2006 fue de unos 8.000 millones de euros, los cuales fueron invertidos principalmente en infraestructura de transporte y en otras inversiones productivas (energía, red eléctrica, fibra óptica, gas, etc.). La mayor parte de los fondos del Objetivo 1 inicial fueron destinados a proyectos de infraestructura y a la construcción de autopistas entre las principales ciudades de la región. En el período financiero 2007-2013, como región elegible dentro del Objetivo de Convergencia, la asistencia financiera europea (incluyendo el Fondo de Cohesión y el Fondo de I+D que es administrado a nivel nacional) sumará unos 4.000 millones de euros, con una reducción del $20 \%$ con respecto al período anterior.

\subsection{El funcionamiento de los Fondos Estructurales: aspectos esenciales de la etapa de programación}

La política regional en España se vio fortalecida a finales de los años ochenta por la interrelación de dos importantes fenómenos políticos: la conclusión del proceso de establecimiento del Estado de las Autonomías y la incorporación a la CE. La descen-

1 Las estadísticas oficiales definitivas no se encontraban disponibles durante la redacción del presente texto por lo que los datos presentados se basan en las últimas estimaciones obtenidas. La misma aclaración se aplica para el caso de Galicia. 
tralización político-territorial y la implementación de una política regional europea activa llevaron a que los niveles de gobierno local, regional, nacional y supra-nacional se involucraran profundamente en la promoción del desarrollo regional (Zaragoza 1990, Correa y Manzanedo 2002).

En relación con la Política de Cohesión europea, y como ocurre con las principales políticas económicas de la UE en general, encontramos dos canales de representación. De un lado, constatamos la voluntad política de los gobiernos de estar activamente presentes en los momentos claves de las políticas europeas. Tal voluntad se refleja en la representación institucional y política de los poderes ejecutivos (nacional y regional), bien directamente, a través de la Oficina del Presidente, o bien vía Ministerio de Asuntos Exteriores o Secretaría de Acción Exterior. Las responsabilidades económicas y de programación se encuentran, en cambio, a cargo de los órganos públicos especializados en la materia, como el Ministerio o Consejería de Economía (nacional y regional). Esta estructura refleja la perspectiva dual clásica de vinculación política y económica (Closa y Heywood, 2004; Cienfuegos, 2000; Molina, 1999; Morata y Muñoz, 1996). Con respecto a las regiones Objetivo 1 (o Convergencia, según la nueva clasificación 2007-2013), es posible afirmar que los fondos estructurales son básicamente canalizados a través del Ministerio de Economía y Hacienda que coopera activamente con las consejerías de economía de las comunidades autónomas².

España ya ha experimentado cuatro períodos financieros de la Política de Cohesión durante los cuales el rol de los gobiernos regionales ha sido reforzado de manera progresiva con respecto a la elaboración de políticas regionales. De hecho, la definición de la estrategia de desarrollo regional es hoy en día dominio de los ejecutivos regionales. La programación estratégica a largo plazo se ha convertido también en una práctica común para cualquier Administración regional española. A pesar de su complejidad, el proceso de la Política de Cohesión europea ha sido incorporado de manera adecuada por las administraciones regionales. Es más, generalmente los programas operativos forman parte de una estrategia regional más amplia y no son considerados únicamente como documentos obligatorios que deben ser entregados -a través del Gobierno central- a la Comisión.

La Figura 2 ilustra el proceso de programación estratégica regional. Los gobiernos autonómicos diseñan planes estratégicos regionales extensivos que contienen las líneas de la política regional a largo plazo. Estos planes estratégicos reciben imputs externos provenientes tanto de la arena nacional como de la política de la UE, que se combinan con las demandas en el nivel regional y local. De esta modo, la estrategia y el conjunto de las intervenciones regionales se encuentran contenidos en el plan estratégico regional.

2 El predominio del Ministerio de Economía en los fondos de las regiones del Objetivo 1 es particularmente evidente para el FEDER. Por supuesto, otros ministerios pueden tener relación con algunas actividades sectoriales financiadas por los fondos estructurales. Por ejemplo, el Ministerio de Trabajo es un importante actor en la administración del FSE. 
FIGURA 2

Planificación estratégica regional y coherencia interna/externa

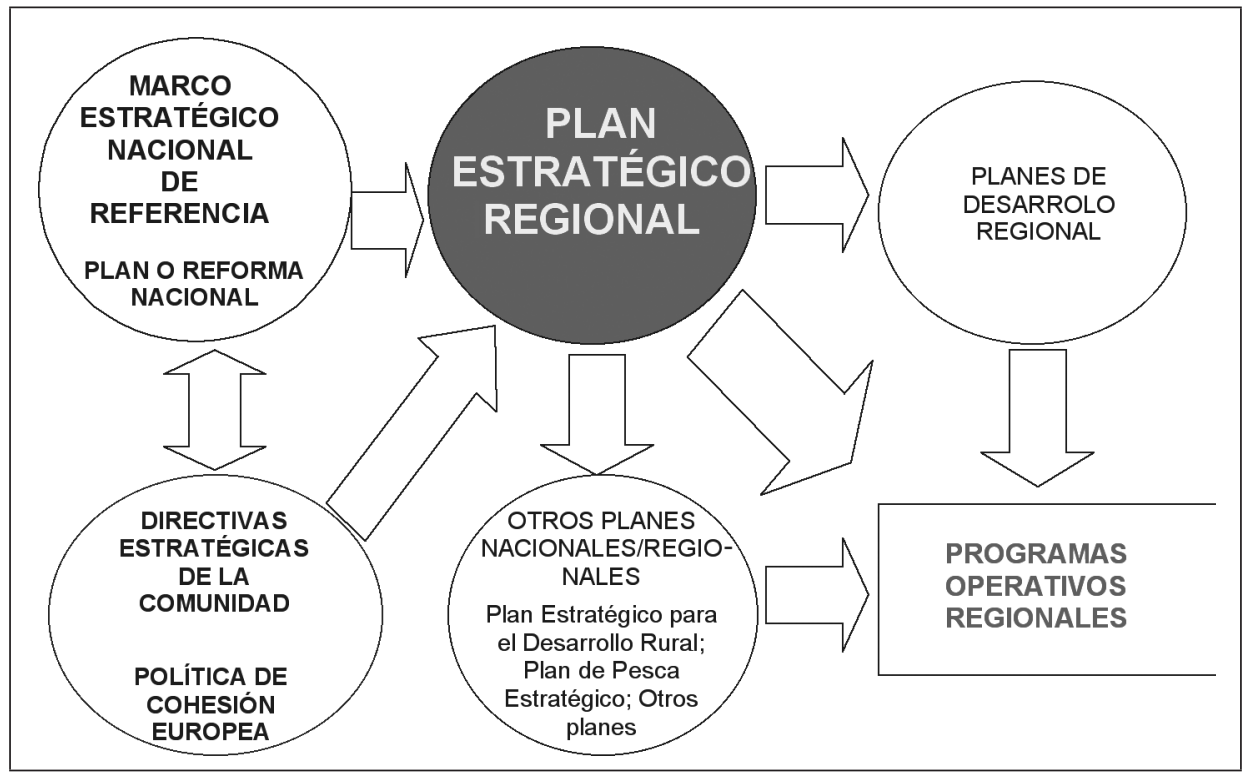

Fuente: Elaboración propia.

Los gobiernos autonómicos pueden financiar sus estrategias de diversas formas. Como es sabido, las limitaciones presupuestarias son frecuentemente severas y los flujos de fondos adicionales son siempre bien recibidos. Además de los ingresos fiscales, las administraciones regionales saben muy bien que pueden recibir transferencias de capital por parte del Estado y de la UE. Pero para acceder a estas fuentes de financiación es un requisito imprescindible la elaboración de algunos documentos formales. Generalmente, la actividad de programación regional comienza diseñando un primer borrador general en el que la administración autonómica describe la estrategia sobre una base plurianual. Concretamente, el documento es elaborado por la Consejería de Economía y luego es sometido a un proceso abierto de debate público y revisión. Hoy en día, las principales consejerías autonómicas, los gobiernos locales, las universidades, el Consejo Económico y Social, los sindicatos, las asociaciones empresariales, organizaciones no gubernamentales y grupos de profesionales, todos ellos pueden participar en el debate presentando sus comentarios sobre el borrador. Finalmente, el plan estratégico regional (revisado) es sujeto a la aprobación del parlamento autonómico. Una vez aprobado, éste representa la estrategia oficial para el desarrollo regional y deviene un documento vinculante para el ejecutivo regional. Posteriomente, el documento es transformado en un Plan Regional de Desarrollo (PRD), es decir, un documento que contempla también aquellas intervenciones cofinanciadas por el Estado y la UE. Sólo de esta manera la estrategia de desarrollo regional puede recibir asistencia estructural europea. Finalmente, los programas ope- 
rativos regionales (PORs) definen esta estrategia en términos más operativos. Como resultado, muchas de las intervenciones contenidas en el plan estratégico regional coinciden con aquéllas contenidas en los programas operativos regionales.

FIGURA 3

Planificación estratégica regional y programas operativos

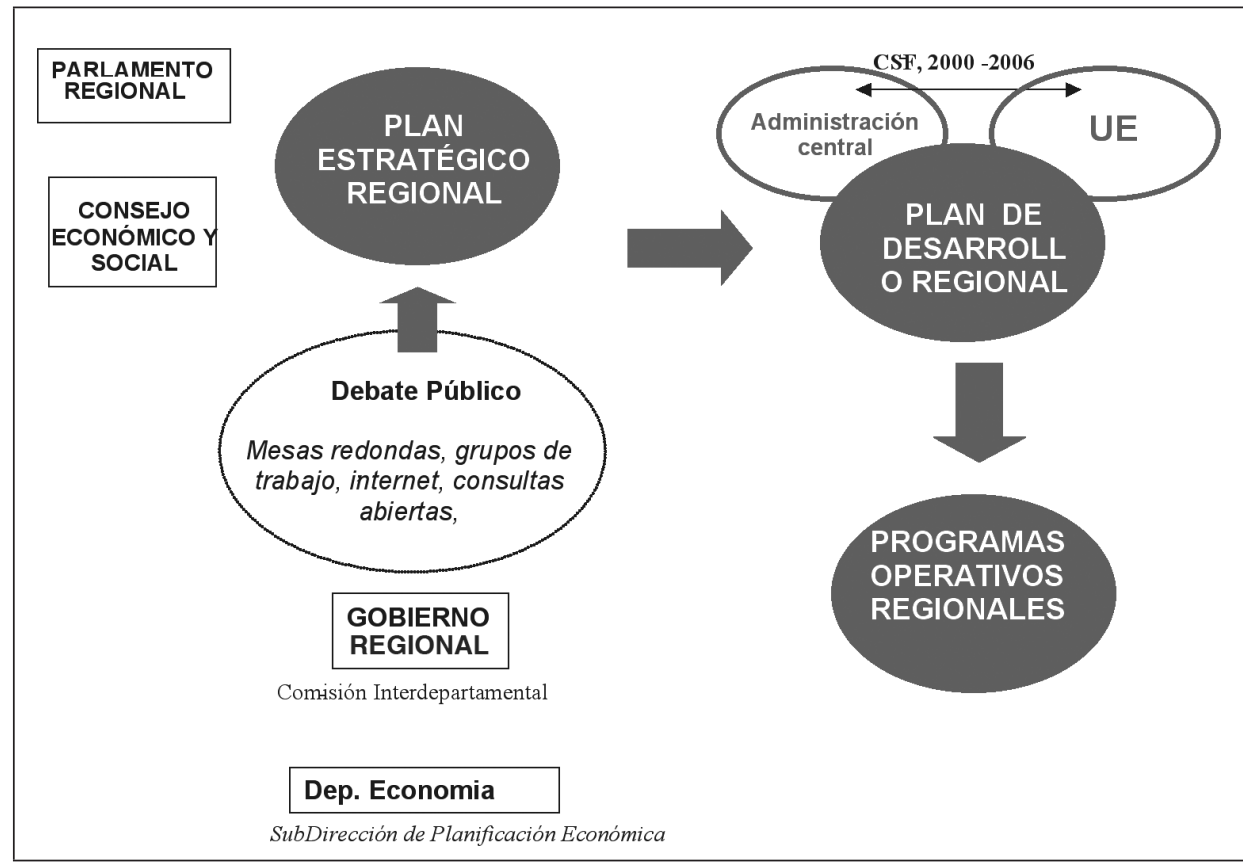

Fuente: Elaboración propia.

Aunque los principales actores de la sociedad civil participan en el debate sobre los documentos estratégicos regionales, la efectividad de la participación puede diferir. Una de las principales preocupaciones que surge de las experiencias previas de programación hace referencia al valor real de la participación de estos actores en el diseño de la estrategia regional. Sin embargo, las sucesivas experiencias de programación regional han conducido progresivamente a una participación más efectiva de los actores sociales. A lo largo de los años, tanto en Galicia como en Murcia los gobiernos autonómicos han ampliado los mecanismos formales de participación. Entre los principales mecanismos utilizados encontramos las entrevistas abiertas con expertos regionales, mesas redondas y grupos de trabajo sobre sectores estratégicos y temas transversales, grupos de discusión, y el lanzamiento de una página web donde los ciudadanos pueden expresar sus opiniones respecto al plan. El Gobierno regional de 94 Murcia, por ejemplo, presentó en julio de 2004 una iniciativa muy ambiciosa, denominada Horizonte 2010, a través de la cual solicitó a todos los ciudadanos que participa- 
ran, de manera personal o colectivamente, en la definición de las prioridades de la política para el desarrollo social y económico de la Región. El resultado concreto de la iniciativa fue el PERM 2007-2013, el Plan Estratégico para la Región de Murcia. Es importante destacar que los dos programas operativos regionales (FEDER y FSE) para el período 2007-2013 fueron extraídos directamente del PERM 2007-2013. Un proceso similar tuvo lugar en Galicia donde el nuevo plan estratégico fue denominado MECEGA 2007-2013 (Marco estratégico de convergencia económica de Galicia, 2007-2013). De acuerdo con este plan se diseñaron los dos programas operativos (FEDER Y FSE) para Galicia.

\section{COMPARACIÓN DE LAS REDES DE POLÍTICAS EN GALICIA Y MURCIA}

La reforma de los Fondos Estructurales, en 1988, introdujo cuatro principios básicos para la Política de Cohesión europea: la programación, la concentración, la adicionalidad, y, en especial, el "partenariado". Este último principio es considerado el principal mecanismo para la implicación de las policy networks en la elaboración de políticas públicas europeas. En particular, el principio de partenariado institucionaliza el contacto directo entre la Comisión y actores gubernamentales no centrales, particularmente autoridades regionales y locales, empresarios y grupos de acción locales (Thielemann 1998:19). La construcción de la gobernanza multinivel en Europa se ha sustentado ampliamente en la aplicación de este principio que, por ello, resulta un elemento clave de la europeización de la política regional en los estados miembros de la UE (Leonardi 2005).

Por una parte, el concepto de "policy network" se refiere a una estructura o complejo de organizaciones conectadas entre sí por relaciones de interdependencia, que abarca autoridades de diferentes niveles de gobierno así como a actores públicos y no públicos (Marsh y Rhodes 1992: 13). El enfoque centrado en la observación de las redes de políticas sirve bastante bien para analizar las variaciones en los modelos de intermediación de intereses y, por tanto, ayuda a trazar las relaciones entre actores públicos y privados (Jordana 1995). En particular, el estudio de las redes de políticas resulta extremadamente útil para reconocer la importancia de los actores no estatales en la elaboración de las políticas europeas.

Por otra parte, es posible distinguir diferentes tipos de policy network implicados en diferentes etapas del proceso de las políticas públicas: desde el extremo de comunidades políticas estrechamente urdidas (policy communities) hasta el opuesto de redes de grupos de presión (issue network, lobbying network, exchange network) (Marsh 1998, Marsh y Rhodes 1992, Ward y Williams 1997). Mientras que la categoría de policy communities describe un tipo de red dominada claramente por los actores gubernamentales (de participación restringida, valores comúnes compartidos, perdurable y mutuamente beneficiosa para todos), hasta el punto de que puede referirse a un modelo de relaciones inter-gubernamentales, en cambio, la categoría de lobbyng network o issue network se refiere más bien a amplias redes configuradas por actores cuya participación puede ser inestable, que intervienen mediante relaciones 
básicamente de consulta, donde el consenso es limitado y, por tanto, son más frecuentes las relaciones de competencia y conflicto.

La observación y análisis de las policy network establecidas en la etapa de programación de la política regional 2007-2013, en la Región de Murcia y en Galicia, sirven para identificar la distribución de poder, influencia y estatus entre los diversos intereses particulares vinculados al área específica de la política regional. La aplicación del principio de partenariado puede producir diversos tipos de policy networks, lo cual dependerá tanto de la acción estratégica de los actores que participan en la red y de la distribución de recursos de poder, estatus e influencia que se produce entre ellos, así como de las reglas institucionales y propias características de la estructura social regional (especialmente sus recursos de capital social).

\subsection{Identificación de la centralidad en las redes regionales}

En este apartado nos proponemos comparar la estructura de las redes de política pública regional en ambas regiones, utilizando datos relacionales obtenidos en las entrevistas realizadas. Dichas entrevistas, efectuadas en los meses de marzo y abril de 2007 y conducidas personalmente, se desarrollaron de acuerdo con un guión y un cuestionario diseñado específicamente para la obtención de datos relacionares y la medición de opiniones y actitudes de los individuos en el papel de representantes de la organización (Oppenheim 1992).

Desde una perspectiva general, las redes de política son bastante similares en ambas regiones. Al observar las redes basadas en los contactos declarados entre los actores, encontramos que las mismas son relativamente densas y que todos los nodos forman un único bloque. El grado de densidad es bastante similar para ambas redes de contactos explícitos entre los actores. Galicia tiene una densidad de 3,263 (DE de 1.21) y Murcia una densidad de 3,291 (DE de 1.59). Sin embargo, surgen diferencias significativas al comparar con detalle ambas redes.

Hemos calculado el grado de centralidad de cada actor (los vínculos hacia adentro y hacia afuera del vértice) tomando en cuenta las respuestas de los actores sobre la frecuencia de los contactos entre ellos ${ }^{3}$. Las diferencias entre los vínculos hacia adentro y hacia afuera son algo desconcertantes: las matrices no son perfectamente simétricas, lo cual significa que algunos actores identifican contactos cuya contraparte no identifica. Además, la intensidad de los contactos es valorada de forma muy diferente entre pares de actores. Una primera aproximación a estas diferencias se encuentra en las Tablas $1 \mathrm{a}$ y $1 \mathrm{~b}^{4}$ en la que presentamos el grado de centralidad de cada actor, desde su perspectiva y desde la perspectiva de los otros.

3 Como los datos son valorados, los grados (hacia adentro y hacia afuera) se componen de las sumas de los valores de los vínculos recibidos por un determinado vértice (grado entrante) y el número de los vínculos originados en el mismo vértice (grado saliente).

$4 \quad$ Para el significado de los acrónimos de las Tablas 1a y 1b véase el anexo al final del artículo. 
En Galicia, los actores gubernamentales tienen una mayor centralidad que los actores no gubernamentales. Sin embargo, la centralidad percibida externamente es menor que la percibida por ellos mismos, incluso existen algunos casos que muestran una gran discrepancia, lo que significa que probablemente muchos actores no valoran tales contactos. En el caso de los actores no gubernamentales encontramos también la autopercepción de un alto grado de centralidad que no se corresponde con las visiones de otros actores pertenecientes a la red (claros ejemplos de ello serían el sindicato Comisiones Obreras y la Universidad de Santiago).

\section{TABLA 1a}

Grado de centralidad de los actores. Galicia

\begin{tabular}{|c|c|c|}
\hline & Grado entrante & Grado saliente \\
\hline 1. ConsPres & 17.000 & 37.000 \\
\hline 2. Fund G-E & 21.000 & 17.000 \\
\hline 3. ConsEco & 37.000 & 42.000 \\
\hline 4. ConsInov & 27.000 & 30.000 \\
\hline 5. ConsTra & 32.000 & 28.000 \\
\hline 6. ConsEdu & 10.000 & 11.000 \\
\hline 7. DipACo & 5.000 & 8.000 \\
\hline 8. FEGAMP & 3.000 & 0.000 \\
\hline 9. ZonaFra & 6.000 & 3.000 \\
\hline 10. CCOO & 21.000 & 1.000 \\
\hline 11. UGT & 17.000 & 25.000 \\
\hline 12. CIG & 16.000 & 31.000 \\
\hline 13. CEG & 19.000 & 22.000 \\
\hline 14. IGAPE & 26.000 & 6.000 \\
\hline 15. EixoAtl & 6.000 & 1.000 \\
\hline 16. IGADI & 14.000 & 6.000 \\
\hline 17. USC & 24.000 & 0.000 \\
\hline 18. CES-G & 12.000 & 33.000 \\
\hline 19. Cámara & 2.000 & 14.000 \\
\hline Media & 16.579 & 16.579 \\
\hline Desv.Est & 9.675 & 13.585 \\
\hline
\end{tabular}

Fuente: Elaboración propia. 
TABLA 1b

Grado de centralidad de los actores. Murcia

\begin{tabular}{|c|c|c|}
\hline & Grado entrante & Grado saliente \\
\hline 1. DelegGob & 8.000 & 10.000 \\
\hline 2. ConsPres & 37.000 & 25.000 \\
\hline 3. RepBrus & 9.000 & 0.000 \\
\hline 4. ConsEco & 42.000 & 28.000 \\
\hline 5. ConsInd & 31.000 & 19.000 \\
\hline 6. ConsTrab & 22.000 & 16.000 \\
\hline 7. ConsAgri & 21.000 & 30.000 \\
\hline 8. INFO & 37.000 & 36.000 \\
\hline 9. FedMuni & 10.000 & 4.000 \\
\hline 10. AyuMur & 12.000 & 4.000 \\
\hline 11. Camara & 11.000 & 27.000 \\
\hline 12. $\mathrm{CCOO}$ & 20.000 & 18.000 \\
\hline 13. UGT & 22.000 & 35.000 \\
\hline 14. CROEM & 31.000 & 19.000 \\
\hline 15. InstMuj & 8.000 & 43.000 \\
\hline 16. AMUSAL & 1.000 & 6.000 \\
\hline 17. UnivM & 25.000 & 7.000 \\
\hline 18. UnivPC & 15.000 & 27.000 \\
\hline 19. ANSE & 7.000 & 6.000 \\
\hline 20. Foro & 0.000 & 9.000 \\
\hline Media & 18.450 & 18.450 \\
\hline Desv. Est & 12.039 & 12.176 \\
\hline
\end{tabular}

Fuente: Elaboración propia.

En el caso de Murcia, los actores gubernamentales también muestran una mayor centralidad que los actores no gubernamentales, aunque las diferencias entre ambos no son tan amplias. Al mismo tiempo, no hay un desequilibrio tan marcado entre los contactos tal como son percibidos por otros ("grado entrante") y como son identificados por cada actor ("grado saliente"), aunque sí existe algún caso de gran desequi98 librio (como el del Instituto de la Mujer). 
Al comparar ambas regiones, encontramos una mayor intensidad de los contactos en Murcia que en Galicia (media de 18.45 contra 16.58), al tiempo que las diferencias entre "grado entrante" y "grado saliente" son menos importantes en Murcia (que muestra un desvío estándar más parecido). Además, los actores sociales y económicos en Murcia muestran un mayor nivel de centralidad que en Galicia -aunque la diferencia no es muy importante globalmente.

A fin de identificar la estructura básica de la red, utilizamos la pregunta sobre los contactos entre los miembros de la misma (excluyendo la medida de las frecuencias). Hemos convertido los datos en una red binaria, para evitar datos valorados, y encontramos que el índice de centralización de la red (Freeman Degree) para Galicia era de $51.85 \%$, mientras que en el caso de Murcia era de $39.89 \%$. De esta manera, como la diferencia entre ambos índices es bastante amplia, podemos inferir que la red de Galicia está mucho más centralizada que la de Murcia. En particular, como hemos observado anteriormente, las distintas unidades gubernamentales en Galicia muestran una posición central altamente relevante en la red, de manera opuesta al caso de Murcia en donde las unidades gubernamentales no son tan centrales, siendo la única excepción la Consejería de Economía, el actor con el mayor nivel de centralidad en la red.

Teniendo en cuenta los cliques de tres miembros, encontramos 27 cliques en Murcia y 22 en Galicia. Es posible observar diferencias significativas entre las regiones en relación con la concentración de los cliques y el rol de los distintos actores que forman parte de estos cliques. Mientras que en la Región de Murcia los actores que integran mayor cantidad de cliques son principalmente no gubernamentales, en Galicia ocurre lo contrario, los actores gubernamentales son quienes tienen una mayor preeminencia entre los miembros de la red. En otras palabras, podríamos esperar que en Galicia el rol de los actores gubernamentales en la formación de los cliques sea más relevante que en el caso de Murcia, en donde muchos de los cliques creados se encuentran compuestos sólo por actores no gubernamentales. Estas diferencias sugieren que las formas en que se desarrolla la acción colectiva en torno a la política regional implican una mayor presencia de, e interacción entre, los actores privados en la Región de Murcia que en Galicia, lo cual presupone unas condiciones de capital social más favorables para la acción colectiva y la participación política entre los actores que participan en la red de política regional murciana.

Las Figuras 4.1 y 4.2 miden el grado de intermediación entre los actores de la red de política de cada región a partir de la información relativa a la frecuencia de los contactos. Esta es una medida de la centralidad de los actores que apunta a identificar cuántas veces otros actores contactan a un actor particular para acceder a un tercero. De esta manera, podríamos sugerir que el grado de intermediación mide las posiciones estratégicas de los actores dentro de la red. Teniendo en cuenta estas figuras podemos identificar la gran importancia que tiene la Consejería de Economía en ambas regiones. Ésta resulta un actor clave en ambos casos, mostrando una posición relevante en la red. Al ampliar la perspectiva, podemos observar que la Presidencia también es un actor relativamente central en ambas regiones. En el caso de Gali- 
cia, observamos una estrecha proximidad entre los siguientes tres actores: Economía, Presidencia y la Fundación Galicia-Europa (formalmente adscrita al último cuerpo); y una estructura similar se observa en la Región de Murcia (ConsEco, ConsPres, INFO). Estos triángulos institucionales aportan evidencia al clásico argumento de la doble perspectiva según el cual el proceso de europeización ha generado dos canales distintos de representación. En la política regional (así como también en el nivel nacional) la representación institucional es delegada en el Gabinete del Presidente mientras que la representación económica es canalizada a través de la Consejería de Economía.

FIGURA 4.1

Murcia: Intermediación de nodos para matriz de contactos "hacia adentro" y "hacia afuera" (medidos por el tamaño del nodo)

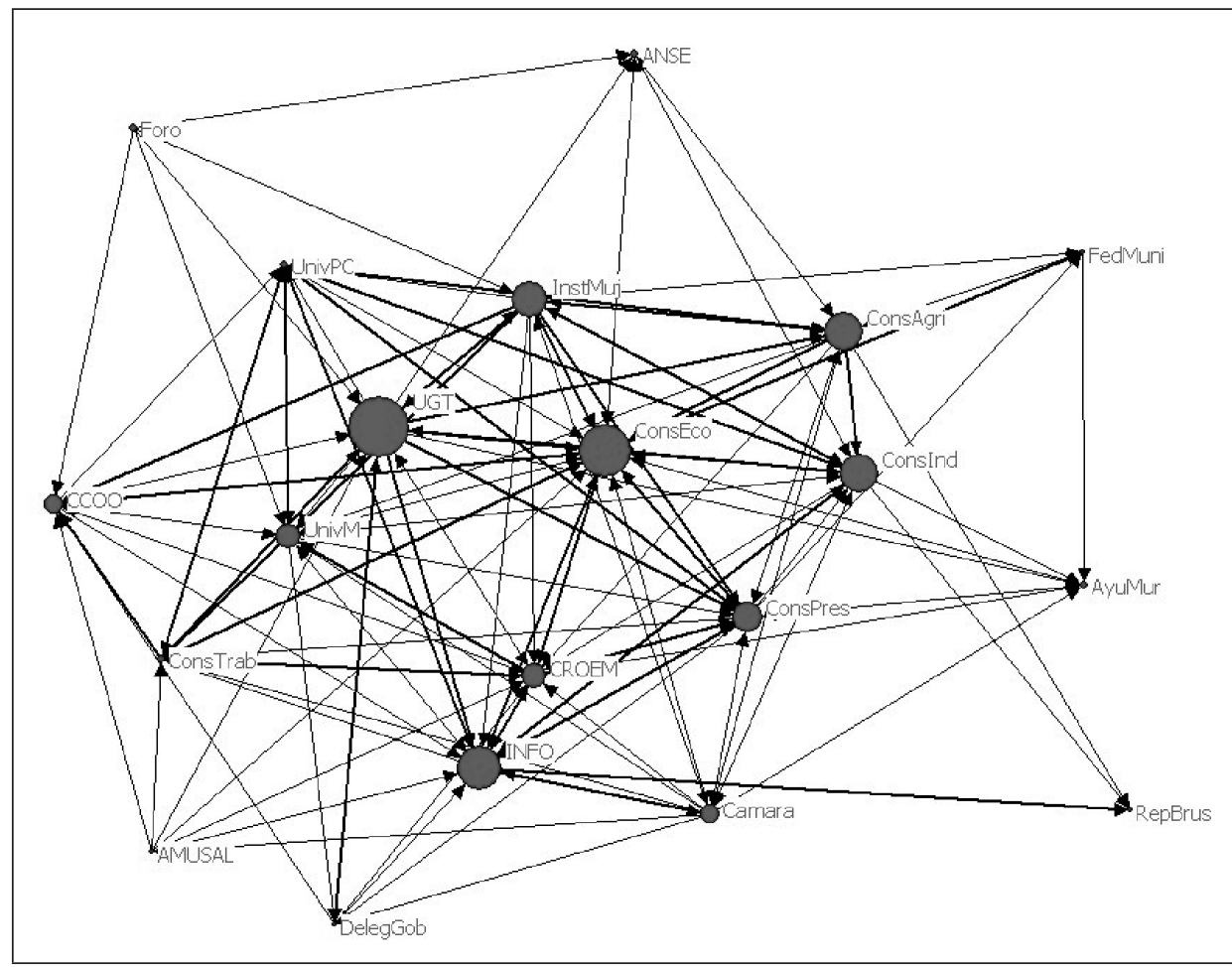

Fuente: Elaboración propia.

No obstante, la comparación de las dos redes muestra una diferencia importante en relación con los actores. En el caso de Murcia, encontramos un número de actores que tiene un grado de intermediación relativamente alto (como por ejemplo, el sindicato de la UGT, otras consejerías, y también la agencia pública INFO); 
mientras que en el caso de Galicia, casi todos los demás actores tienen un grado de intermediación bastante reducido. Esta diferencia refuerza la observación de que la Consejería de Economía en Galicia tiene una centralidad relevante y una capacidad estratégica mayor que cualquier otro actor, permitiéndole de esta manera concentrar importantes recursos de poder (lo que no es equivalente para el caso de Murcia).

\section{FIGURA 4.2}

Galicia: Intermediación de nodos para matriz de contactos "hacia adentro" y "hacia afuera" (medidos por el tamaño del nodo)

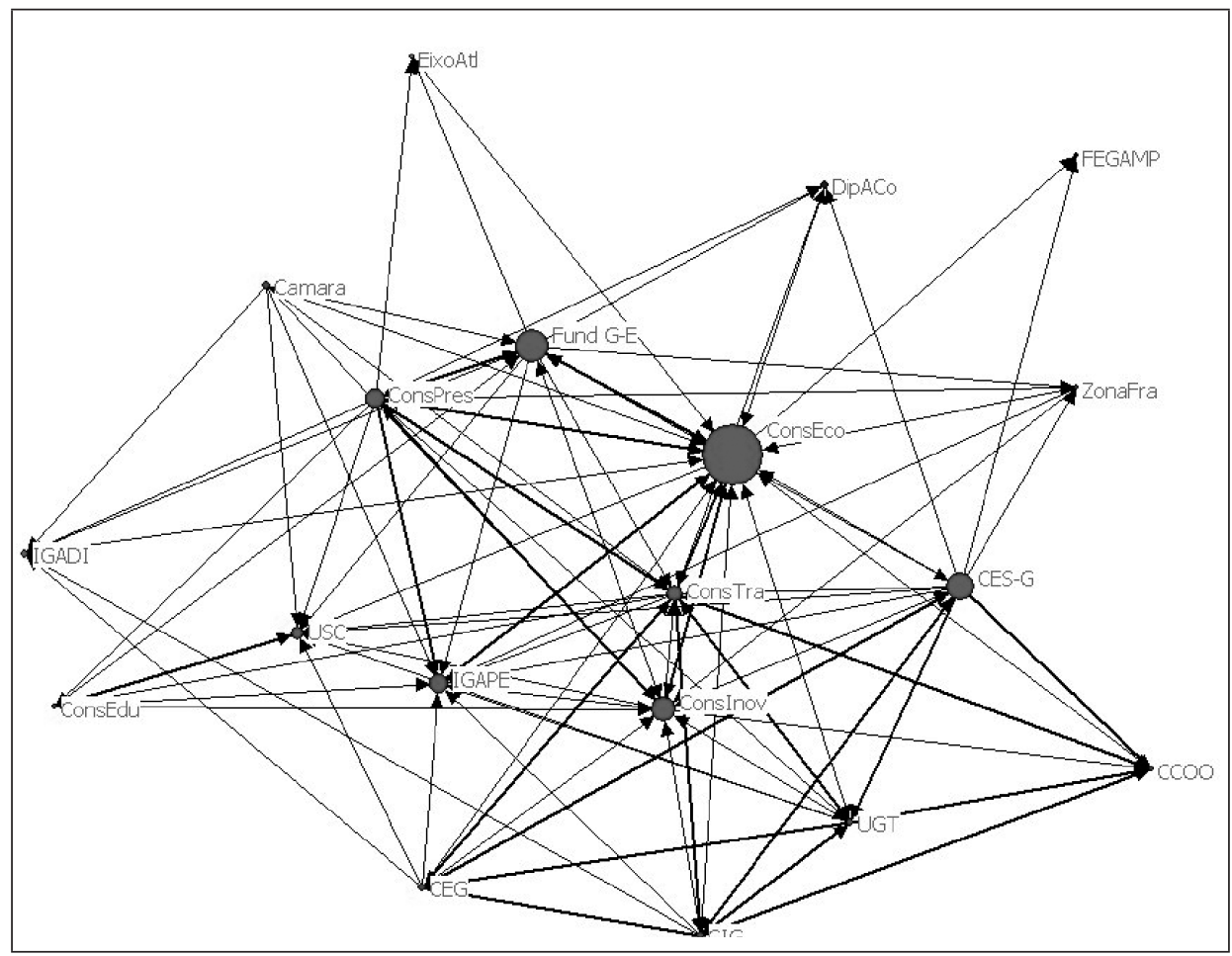

Fuente: Elaboración propia.

Finalmente, es importante señalar que las oficinas de representación regional en Bruselas (Fundación Galicia Europa y la Representación Permanente de la Región de Murcia en Bruselas) pertenecen a la red, pero no tienen posiciones relevantes en la misma. Las instituciones regionales ubicadas en Bruselas pueden transmitir información relevante a la arena regional, pero no participan directamente en actividades de planificación económica y, como ya hemos apuntado, la etapa de programación depende estrictamente de la Consejería de Economía. 
Otra característica configuradora de las redes de política regional se refiere a los receptores de los contactos en cada red. En las Figuras 5.1 y 5.2 observamos que en la Región de Murcia es relativamente alto el número de relaciones iniciadas por la "segunda parte" o contraparte del actor informante de la relación (en lugar de por el mismo actor informante o por una tercera parte), mientras que en Galicia es muy bajo este tipo de iniciación de los contactos declarados. Una estructura menos jerárquica de la red podría explicar esta tendencia para el caso de Murcia, puesto que muchos de los contactos surgen de actores relativamente periféricos hacia actores más centrales en la red.

FIGURA 5.1

Murcia: Receptor de contactos (iniciados por la contraparte)

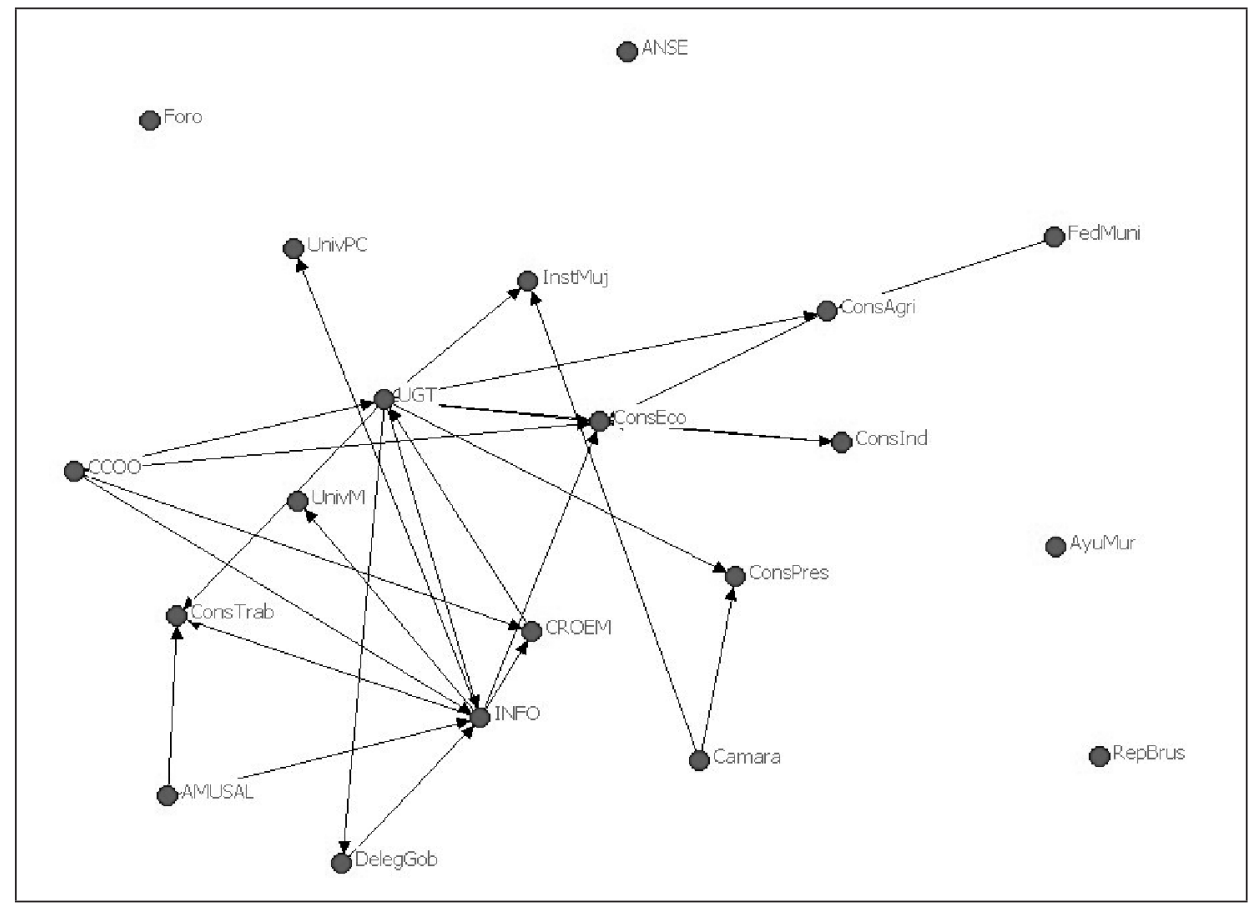

Fuente: Elaboración propia.

Es importante subrayar que las entrevistas se centraron en identificar la estructura de la red formada durante la fase de programación del período financiero 20072013, y en Murcia observamos que los mecanismos de consulta pública promovidos desde el gobierno regional concluyeron en acuerdos que integraron a una pluralidad de actores sobre la base de una mayor igualdad. En primer lugar, Horizonte 2010 y el 102 PERM 2007-2013 fueron oficialmente firmados por el Presidente de la región, los sin- 
dicatos (CC.OO y UGT) y la asociación empresarial regional (CROEM). En segundo lugar, el grado de institucionalización del acuerdo fue fortalecido por la presencia de una Comisión Ejecutiva (presidida por el Presidente de la región con la participación del CC.OO, la UGT y la CROEM) y por la implementación de un comité de seguimiento regional (Comité de Seguimiento del PERM 2007-13), compuesto por las principales consejerías autonómicas (Presidencia, Industria, Trabajo, Economía, Educación, etc.) y también por el INFO, la autoridad medioambiental regional y el Instituto para la Igualdad de Género (Instituto de la Mujer).

FIGURA 5.2

Galicia: Receptor de contactos (iniciados por la contraparte)

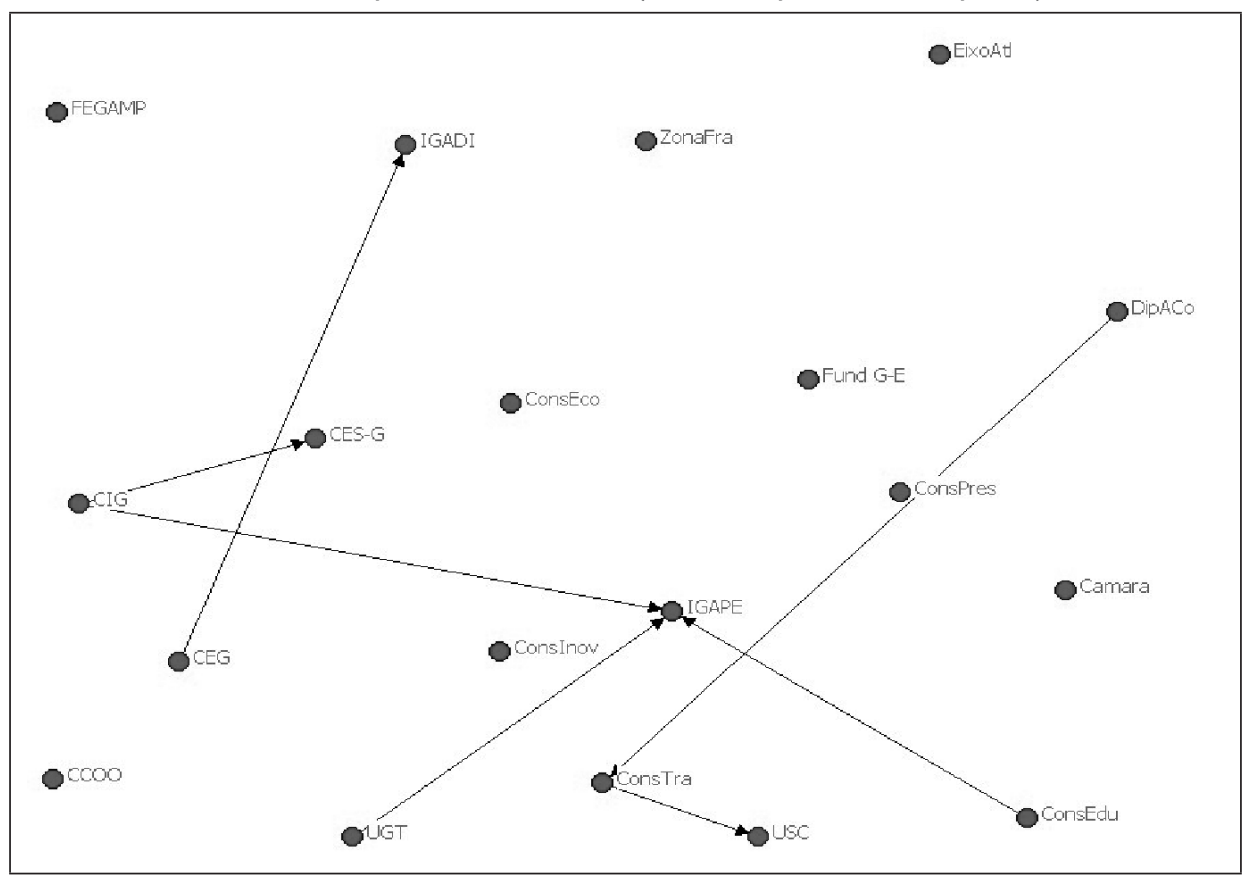

Fuente: Elaboración propia.

\subsection{Las redes de política en acción: las posiciones y percepciones de los actores}

Los conflictos y tensiones entre los actores que pertenecen a la red también muestran distintos patrones cuando se comparan las dos regiones (Figuras $6.1 \mathrm{y}$ 6.2). En el caso de Galicia no hay aparentemente grandes conflictos, siendo la única área de tensión aquélla vinculada a las relaciones entre los sindicatos y las asociaciones de empleadores, que involucra también a algunas consejerías. En cambio, en 
el caso de Murcia las dimensiones del conflicto son más complejas. Encontramos más competencia entre los actores no gubernamentales, probablemente basada en las tensiones por controlar la representación de distintas áreas de interés; también existe la tensión tradicional entre los empresarios y los sindicatos, y encontramos cierto nivel de competencia dentro del gobierno, entre diferentes consejerías. En síntesis, en Murcia existe un patrón de tensiones más pluralista, que se extiende en distintas dimensiones, en comparación con Galicia, donde el conflicto es menos visible y se encuentra principalmente concentrado en la tradicional dimensión de trabajo y patronal. Estas diferencias refuerzan las observaciones previas realizadas sobre el grado de centralidad y la participación de los actores en la red de política regional en ambas regiones. En particular, apuntan a que el patrón de policy network configurado en la política regional de Galicia es más próximo al tipo de relaciones inter-gubernamentales y policy community, mientras que en la Región de Murcia se ha configurado una policy network más abierta, pluralista, y por ello más conflictiva.

\section{FIGURA 6.1}

Murcia: Conflictos en la policy network (percepción de relaciones competitivas o conflictivas)

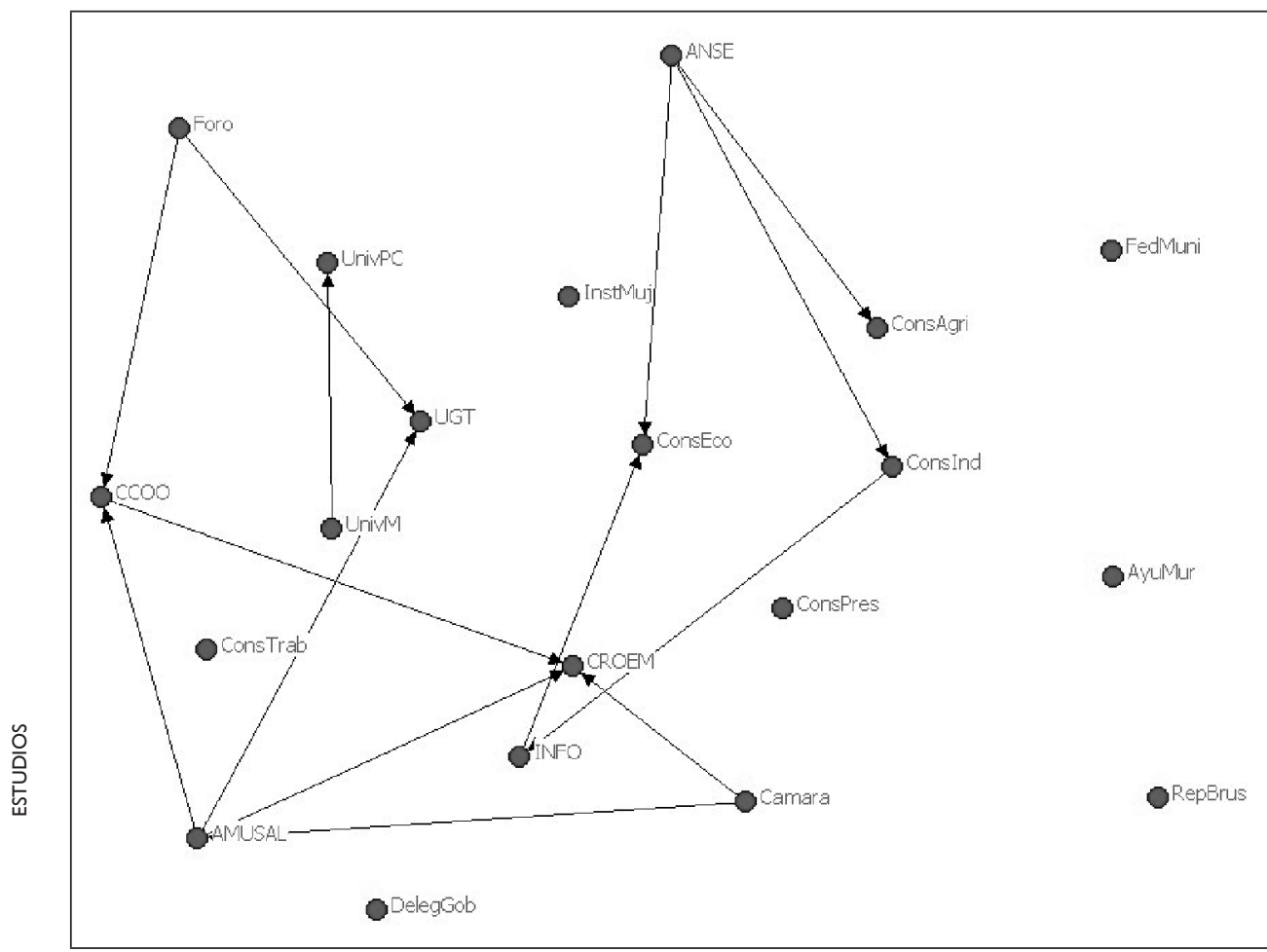

Fuente: Elaboración propia. 
Una diferencia significativa relativa a las posiciones de los actores en cada una de las redes regionales, y que parece estar asociada con la dinámica del conflicto en el seno de cada una de ellas, se refiere al rol jugado por los Consejos Económicos y Sociales (CES) de cada comunidad. Mientras que el Consejo Económico y Social de Galicia es un miembro permanente del comité de seguimiento 2000-2006 POI, el CES de la Región de Murcia no se encuentra formalmente incluido en el comité de seguimiento respectivo. Esta diferencia también se ve reflejada en nuestro análisis de la red, puesto que el CES Murcia se encuentra ausente en el gráfico de dispersión. No obstante, las diferencias deben ser analizadas con cautela dado que pueden generar cierta confusión. En Murcia, las principales organizaciones sociales (CC.OO, UGT y la asociación de empresarios (ROEM) participan directamente en la actividad de programación estratégica que es dirigida por el gobierno regional. En Galicia, por el contrario, al estar la red más centralizada, encontramos la presencia sobresaliente de una organización, el CES, que centraliza ella misma la representación regional de los grupos de intereses sociales y económicos.

\section{FIGURA 6.2}

Galicia: Conflictos en la policy network (percepción de relaciones competitivas o conflictivas)

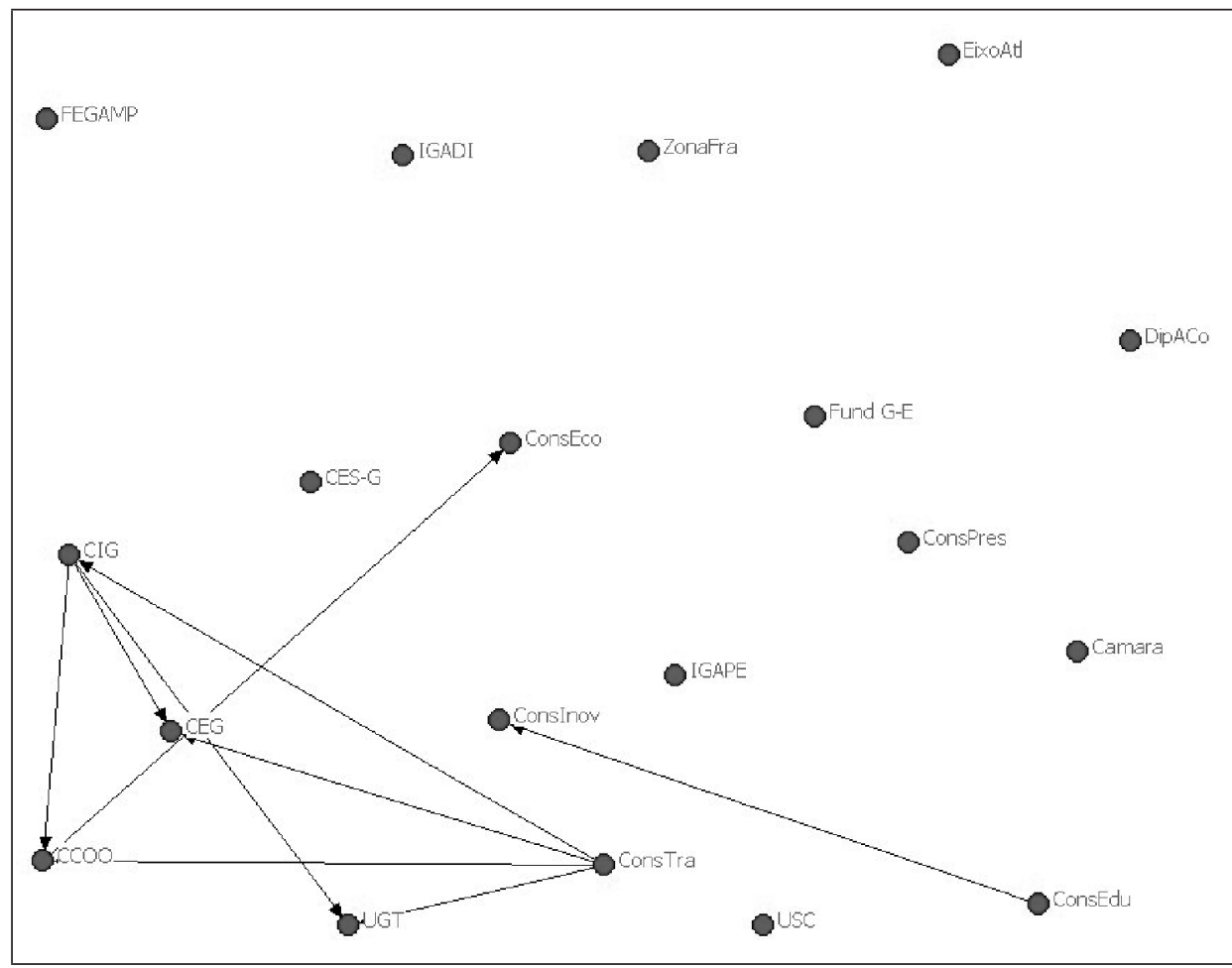

Fuente: Elaboración propia. 
En ambas regiones, parece que un pequeño número de actores concentra las percepciones de muchos otros en lo que respecta a su influencia positiva sobre la formulación de la política regional. Este es el caso de las agencias de desarrollo y de las consejerías de economía. Aunque el rol de las agencias en la etapa de planificación es menos relevante que en la etapa de implementación (en la que generalmente actúan como un organismo intermedio que canaliza la ayuda financiera europea hacia los beneficiarios finales), tanto el INFO como el IGAPE pueden ser considerados actores centrales en este proceso. El Institutio Gallego de Promoción Económica (IGAPE) fue creado en 1993 y directamente vinculado a la Consejería de Economía. Este Instituto brinda información, ayuda y asistencia a negocios en todas las etapas de su desarrollo y tiene varios programas de apoyo a empresas con estrategias para mejorar su rendimiento. El IGA$\mathrm{PE}$, junto con otras instituciones regionales y el gobierno central, es también la fuente central de ayuda a través de nuevos proyectos de inversión viables. Por su parte, el Instituto de Fomento de la Región de Murcia, que forma una parte orgánica de la Consejería de Industria y Medio Ambiente, tiene como principal objetivo fomentar el desarrollo de PYMES por medio de la promoción económica, la búsqueda de inversiones, la eliminación de obstáculos para el desarrollo de negocios y el establecimiento de un ambiente propicio para la competitividad. Desde el período financiero 1989-1994, el INFO es beneficiario de una Subvención Global financiada por el FEDER.

\section{ELABORACIÓN DE POLÍTICAS Y CAPITAL SOCIAL DE LOS ACTORES}

En este apartado examinamos las condiciones de capital social con que cuentan las redes de política regional observadas en Murcia y en Galicia. Aunque existe evidencia empírica que indica un nivel de capital social muy similar en las poblaciones de ambas comunidades, sin embargo, en el colectivo específico de los actores implicados en la red de política regional observamos diferencias significativas entre las dos regiones. Hay que recordar que el capital social no pertenece a un individuo o a un grupo: se trata más bien de un concepto relacional que se refiere a "las características de la organización social, tales como la confianza, las normas y las redes que pueden mejorar la eficiencia de la sociedad al facilitar la acción coordinada" (Putnam 1993: 167). El capital social, como bien público, tiende a ser poco valorado y ofertado por los agentes privados (Herreros 2004), por lo que resulta crucial el papel de las instituciones públicas y, en particular, de las estructuras de elaboración de las políticas públicas a fin de generar y promover el capital social entre los actores involucrados.

\subsection{La distribución regional del capital social en España}

El capital social en España es comparativamente inferior al de otras democracias avanzadas pero similar al de otros países del sur de Europa con quienes comparte el denominado "síndrome meridional" (Maravall 1995, Monterio, Font y Torcal 2006). Como en otros países europeos, el capital social en España también se distribuye de manera des-

106 igual entre las comunidades autónomas. En primer lugar, los estudios sobre capital social 
han mostrado una fuerte asociación positiva entre el nivel regional de capital social ${ }^{5}$ y el grado de desarrollo socioeconómico (Mota 2000 y 2008): las regiones más ricas y prósperas son las mismas que tienen un grado mayor de capital social, y viceversa. Este resultado coincide con el obtenido por Putnam en su estudio sobre las regiones italianas, lo cual refuerza la tesis de que el capital social favorece el logro económico. Es más, la distribución regional de capital social en España configura un mapa donde el valle del río Ebro y la costa mediterránea (además de la Comunidad de Madrid) albergan las regiones con una mayor reserva de capital social, las cuales también son las comunidades más prósperas. De esta manera, los índices de capital social en las dos regiones de nuestro estudio se sitúan entre los más bajos de las comunidades autónomas: en concreto, en una escala de 0 a 1, la Región de Murcia manifiesta un nivel de capital social de 0,19 y Galicia de 0,18.

Por tanto, a principios de la década, Murcia y Galicia presentaban niveles muy homogéneos de capital social, considerando a la población regional en su conjunto, hallándose ambas comunidades entre los territorios de España menos favorecidos en términos de capital social. A partir de aquí analizamos las variables relativas al capital social de los principales actores involucrados en el proceso de elaboración de la política regional. El objetivo que nos proponemos es analizar hasta qué punto las similitudes a nivel agregado entre las dos regiones se confirman en las actitudes de los actores implicados en la red de la política regional, así como también en sus opiniones y creencias sobre la política de desarrollo regional.

\subsection{El capital social y la Política de Cohesión Europea}

En nuestras entrevistas a los actores que participan en la red de política regional incluimos varias preguntas específicas a fin de medir sus características relativas al capital social. Es importante tener en cuentra que nuestros datos no provienen de una muestra estadísticamente representativa, por lo que de ninguna manera es representativa de la población regional en su totalidad ni de la elite política regional. Las respuestas que obtuvimos nos permiten medir diversas dimensiones del capital social exclusivamente para esos individuos. Se debe recordar que fueron seleccionados de acuerdo a la "identificación de posición" y la "identificación de reputación", de manera que representan suficientemente bien a los actores implicados en el proceso de programación de la política regional.

En primer lugar, consideramos el nivel de confianza. La confianza constituye el elemento más importante del capital social. Es el mecanismo conceptual clave para resolver la incertidumbre en las relaciones entre iguales y facilitar la acción colectiva. Actual-

5 La medición del capital social regional realizado por Mota (2000 y 2008,) implica tres dimensiones analíticas y un conjunto de variables: i) Actitudes y comportamientos cívicos: el interés de los ciudadanos en la política regional, la información sobre las actividades del gobierno regional, y el círculo de lectores de diarios; ii) Características de la estructura asociativa (por un lado, la proporción de asociaciones profesionales y económicas sobre el total de asociaciones, y por otro lado, la proporción de asociaciones filantrópicas y de asistencia social sobre el total de asociaciones); iii) la afiliación voluntaria a asociaciones (partidos políticos, sindicatos, clubes deportivos y de recreación, ayuda mutua, etc.). 
mente, la confianza es generalmente considerada un requisito necesario para el éxito competitivo y la mejora del rendimiento institucional. En particular, hemos considerado dos dimensiones de la confianza: la confianza institucional y la confianza social.

En primer lugar, la confianza institucional se refiere al grado de confianza en las instituciones públicas que, medida en una escala de 0 a 1, muestra una puntuación más alta entre los actores de Murcia $(0,69)$ que los de Galicia $(0,59)$. En ambas regiones, las instituciones que administran los fondos de la UE en España y los funcionarios regionales son las instituciones más confiables, sobre todo en comparación con las instituciones locales, pero también en relación con el nivel regional y central de gobierno (Figura 7). Estos resultados, similares en ambas regiones, parecen indicar que se trata de una confianza pública basada esencialmente en la competencia de las instituciones y actores públicos para actuar con eficacia, antes que en la creencia en la voluntad política de los mismos para responder a las demandas políticas. De alguna manera, la confianza pública que manifiestan los actores implicados en la redes de política regional parece reflejar la típica legitimidad por resultado de las políticas de la Unión Europea (Scharpf 1999: 38).

FIGURA 7

Confianza en instituciones públicas en Murcia y Galicia (escala 0-10)

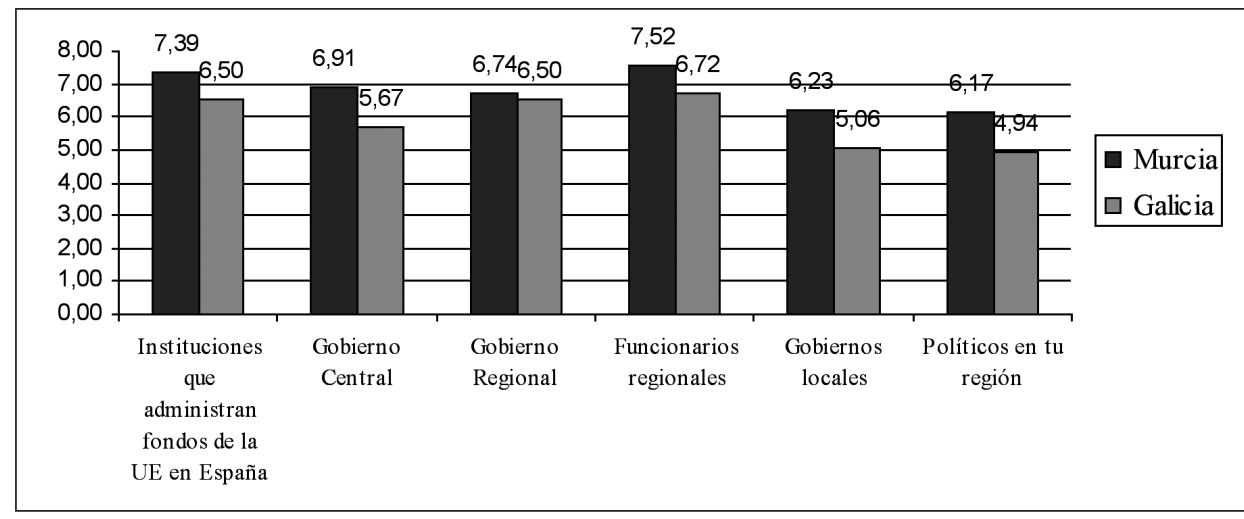

Fuente: Elaboración propia.

En segundo lugar, la confianza social se refiere a la confianza generalizada entre los individuos y las normas sociales de interacción entre ellos. Las tres variables que integran la confianza social (confianza, altruismo y honestidad) muestran valores más altos en Murcia que en Galicia (Figura 8). La construcción de un índice aditivo de la confianza social permite resumir estos resultados en una escala de 0 a 1, en la que los actores de la policy network de la Región de Murcia se sitúan en el 0,66 y su contraparte en Galicia se ubica en el 0,62. La menor diferencia que separa a los actores de la política regional de sendas comunidades en la dimensión de la confianza social, respecto a la confianza institucional, resulta congruente con el nivel de capital social prácticamente 108 idéntico observado en la población de estas comunidades autónomas. 


\section{FIGURA 8}

Nivel de confianza social en Murcia y Galicia

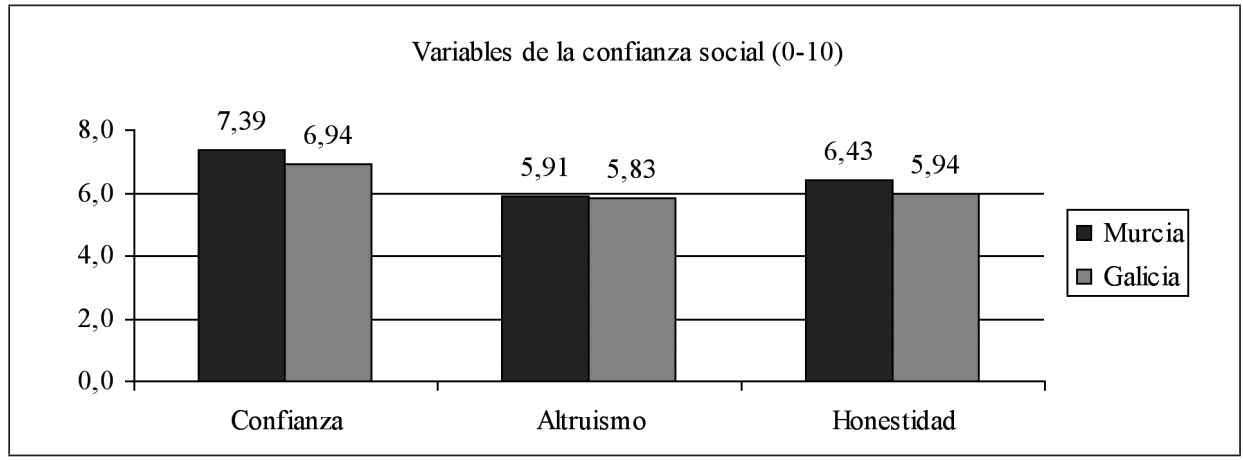

Fuente: Elaboración propia.

\section{FIGURA 9}

Opiniones políticas y sociales en Murcia y Galicia (grado de acuerdo 0-1)

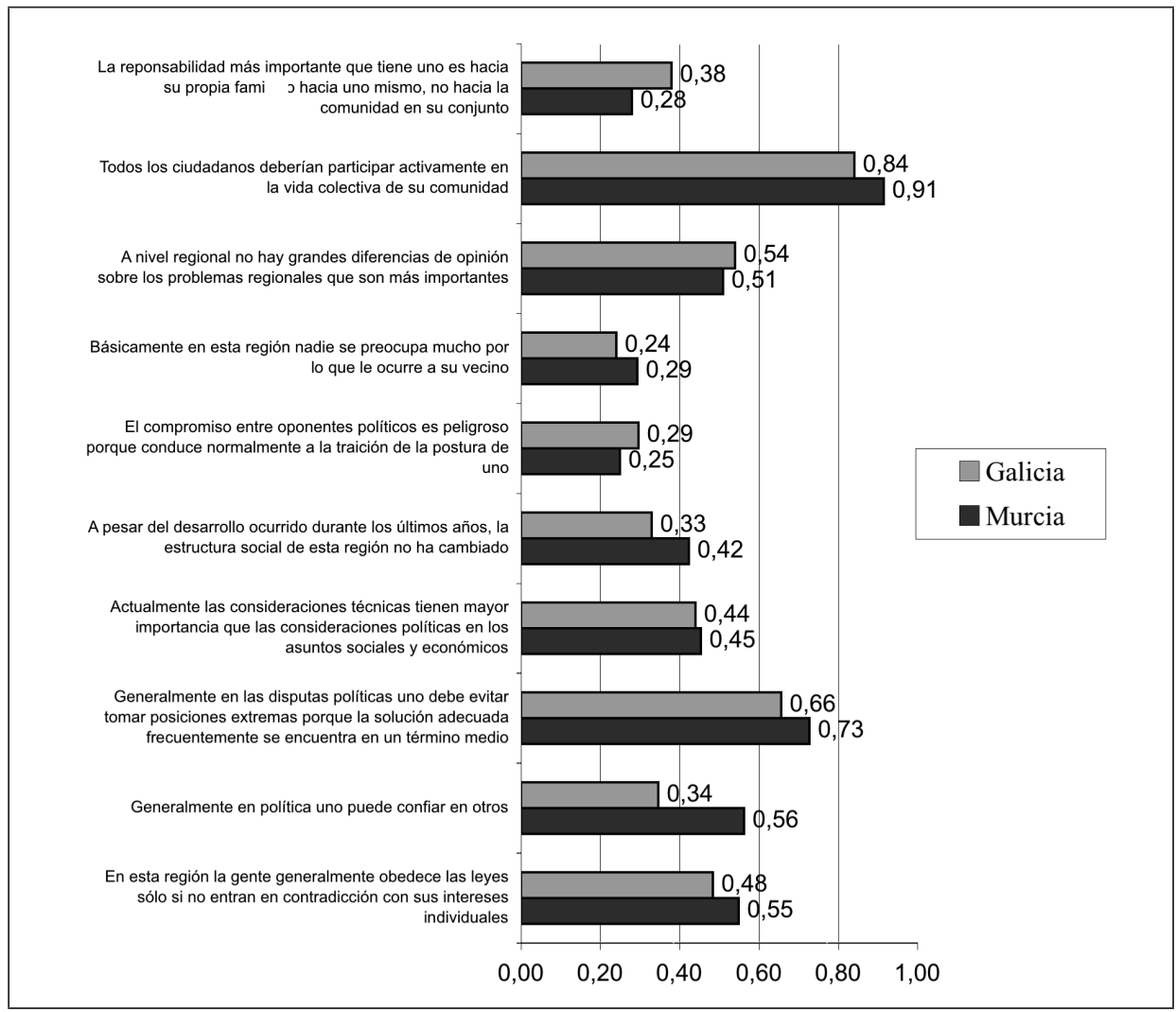

Fuente: Elaboración propia. 
Las actitudes, los valores sociales y las orientaciones vinculadas a la política y a la sociedad definen la cultura política de una comunidad y permiten identificar al mismo tiempo las formas que adopta el capital social. Preguntamos a los actores regionales sobre su grado de acuerdo con distintas opiniones referentes a la política y la sociedad en su propia región. Los valores medios (que oscilan entre o y 1) se muestran en la Figura 9. Resulta interesante observar cómo los entrevistados de las dos regiones describen escenarios regionales diversos. Dado el pequeño tamaño de nuestra muestra es difícil inferir alguna conclusión, aunque distintas opiniones parecen apoyar la existencia en cada región de un modo específico de percibir la confianza social y los valores, así como un patrón predominante de interacción.

Al aplicar un análisis factorial para reducir los resultados obtenidos, obtenemos que estas creencias y opiniones se combinan de manera que proporcionan tres dimensiones subyacentes. El tercer componente (que explica el 14,5\% de la varianza) puede ser considerado como una variable proxy del capital social asociado a la noción de "nexo" (bonding social capital), el cual se refiere a las fortalezas internas de los grupos sociales primarios, como las familias, clanes y los vecinos de una comunidad en defensa de los intereses de grupo y, en particular, como mecanismos para afrontar vicisitudes por parte de los individuos en momentos de severas dificultades y ante la ausencia de instituciones (Narayan 1999; Putnam 2000). Incluye percepciones sobre la ausencia de solidaridad ("Ios vecinos no se preocupan unos por otros", factor loading 0,807), la persistencia de estructuras sociales tradicionales "pese al desarrollo experimentado en los últimos años" (factor loading 0,568) y orientaciones opuestas al compromiso político "que conduciría a la traición de una de las partes" (factor loading 0,488). El segundo componente (que explica el $17 \%$ de la varianza) se encuentra asociado a la noción de capital social como "puente" (bridging social capital), el cual se refiere a la capacidad de asociación de una comunidad y se expresa en redes densas de intercambio social. Estas redes son consideradas antagónicas a las fuerzas de "nexo" (Putnam 2000). Este componente incluye actitudes y opiniones que en cierta medida pueden facilitar la cooperación entre los grupos sociales; como la moderación política ("la solución apropiada generalmente se encuentra en el medio", factor 0,688 ), el individualismo ("la responsabilidad primaria de uno es hacia su propia familia o hacia uno mismo", factor 0,738 ) y la relevancia de una perspectiva técnica antes que ideológica ("actualmente las consideraciones técnicas tienen un mayor peso que las consideraciones de tipo político en los asuntos sociales y económicos", factor 0,767). Por último, el primer y más importante componente (que explica casi el 19\% de la varianza total) puede ser asociado a la noción de "vínculo" (linking social capital), que se refiere a determinadas características y mecanismos de la estructura social que permiten que el capital social "puente" o la capacidad de asociación de una comunidad se exprese a través del comportamiento político, de manera que interactúe con las instituciones y contribuya a la producción de bienes y resultados públicos (Cote y Healy 2001; Woolcock 2001). 110 Por tanto, este componente implica confianza dentro de la esfera política ("en 
política generalmente uno puede confiar en otros", factor loading 0,726) y la implicación de los ciudadanos en la política ("todos los ciudadanos deben participar activamente en la vida colectiva de su comunidad”, factor loading 0,741). Es importante resaltar que este tipo de capital social es esencial, en el contexto del desarrollo económico asociado a la gestión de la política de cohesión de la UE, en tanto que provee los vínculos entre las "instituciones" (las estructuras políticas y organizativas oficialmente responsables de llevar a cabo la política) y la "sociedad civil" (los actores regionales económicos y sociales que tienen la capacidad de transformar los programas en formas de desarrollo sostenible).

La Figura 10 representa los valores medios de cada componente del capital social en ambas regiones. En primer lugar, los valores y las percepciones sociales de los actores involucrados en la política regional están relacionados principalmente con la noción de capital social como "vínculo" (linking social capital). Mientras que entre Murcia y Galicia existen niveles bastante similares de capital social como "nexo" (bonding social capital) y especialmente de capital social como "puente" (bridging social capital), la diferencia más notoria entre estas dos regiones se refiere al capital social como "vínculo" (el cual presenta una diferencia superior a diez puntos en la Región de Murcia). De acuerdo con el modelo teórico del capital social, este resultado parece ser coherente con los resultados anteriores, de modo que los valores que apoyan la confianza social, y especialmente la confianza institucional, así como el compromiso cívico, son mayores entre los actores implicados en la política regional de Murcia que en Galicia.

FIGURA 10

Dimensiones del capital social: nexo, puente y vínculo (rango o a 1)

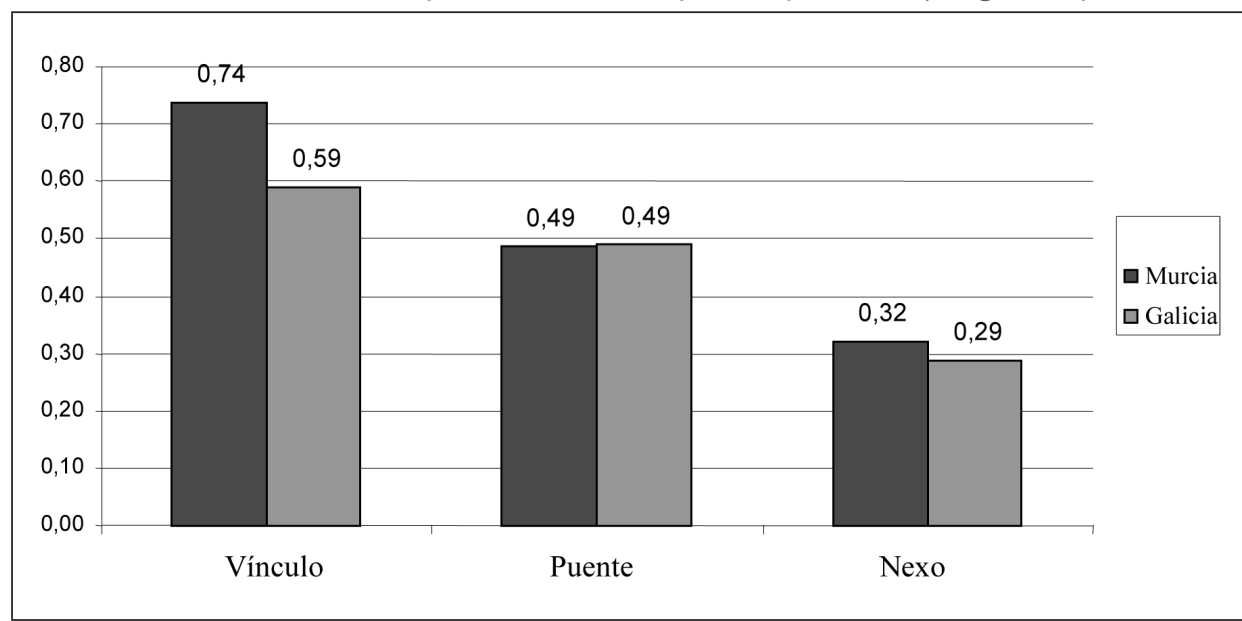

Fuente: Elaboración propia. 


\subsection{Opiniones y percepciones sobre la política regional y la financiación de la UE}

Hemos tratado de examinar el proceso de elaboración de la Política de Cohesión en ambas regiones a través de las opiniones y percepciones de los principales actores participantes. En primer lugar, a la pregunta de cuánta influencia tienen los grupos sociales en el proceso decisorio regional surge una clara división de opiniones entre los entrevistados en la Región de Murcia y en la comunidad gallega. Mientras que el $68 \%$ de los primeros considera que las organizaciones sociales y los grupos de interés regionales ejercen influencia, esta misma opinión no alcanza el 30\% en el caso de los segundos. Por otra parte, más de la mitad de los entrevistados de Galicia considera que las organizaciones sociales tienen escasa influencia en el proceso de toma de decisiones regional. Estas opiniones confirman algunos aspectos de nuestro análisis de red en donde encontramos que ésta es más centralizada (y dominada por instituciones públicas regionales) en Galicia. Por el contrario, la mayor participación activa de grupos de intereses en Murcia, como los sindicatos y las asociaciones empresariales, se refleja en la percepción que tienen los actores sobre la influencia de las organizaciones sociales en la elaboración de la política regional.

FIGURA 11

Formas de participación en la programación de actividades (2007-2013)

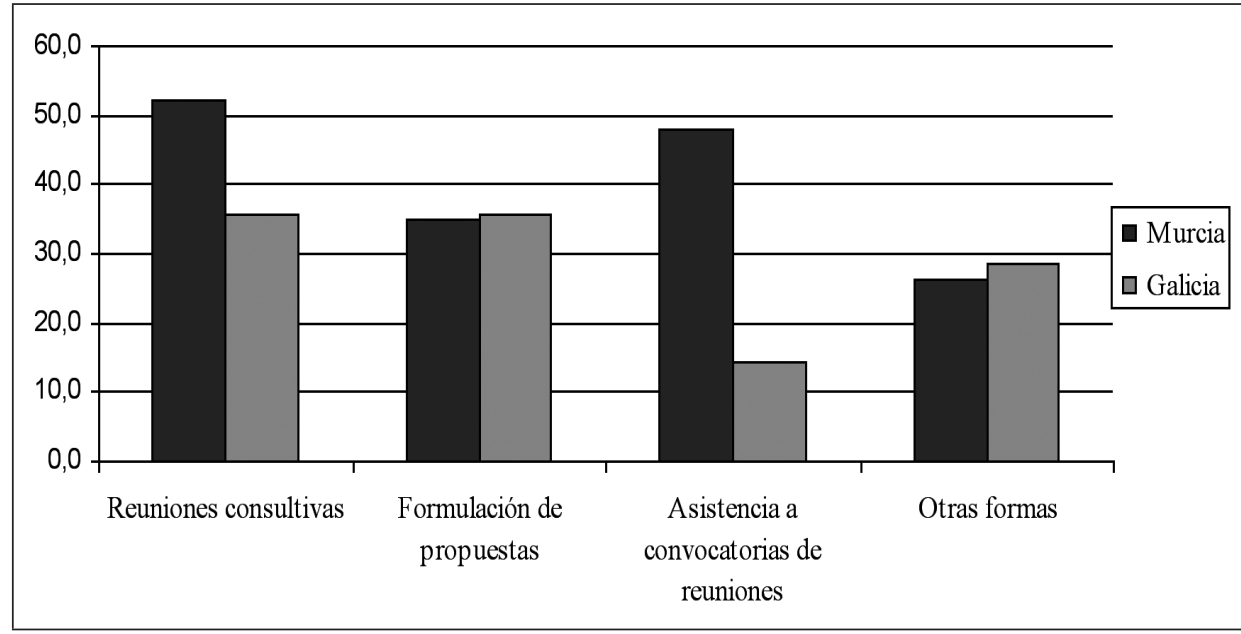

Fuente: Elaboración propia.

La Figura 11 presenta los principales canales de participación a través de los cuales las organizaciones sociales podrían haber participado en la elaboración de la política regional. En términos generales se observa que un mayor número de actores en Murcia que en Galicia afirma haber utilizado varias formas de participación social en

112 la etapa de programación 2007-2013. Las reuniones consultivas y la asistencia a con- 
vocatorias de reuniones representan los mecanismos más utilizados de participación. El resultado es congruente con el proceso que se ha desarrollado en España como consecuencia de la europeización de la política regional. La etapa de programación generalmente contempla algunos procedimientos de consulta a través de los cuales se les pide a los actores sociales que expresen sus opiniones y comentarios sobre la estrategia regional. Sin embargo, la efectividad de la participación de los distintos actores sociales puede ser muy diferente. Las diferencias observadas entre los casos de Murcia y de Galicia indican cuán diferentes pueden ser esas experiencias de participación en la práctica real.

En relación con la naturaleza del conflicto político dentro de la esfera regional, no encontramos variaciones importantes entre las dos regiones. En Galicia y en Murcia el acuerdo político es un bien costoso que no siempre es posible alcanzar por los participantes. Es más, la mitad de los entrevistados en ambas regiones afirman que el compromiso no es frecuentemente utilizado para resolver problemas regionales sustanciales. No obstante, los actores en Murcia perciben que los problemas cruciales son resueltos a través del compromiso de manera más frecuente que su contraparte en Galicia.

\section{FIGURA 12}

Percepciones del estilo político regional (0-10)

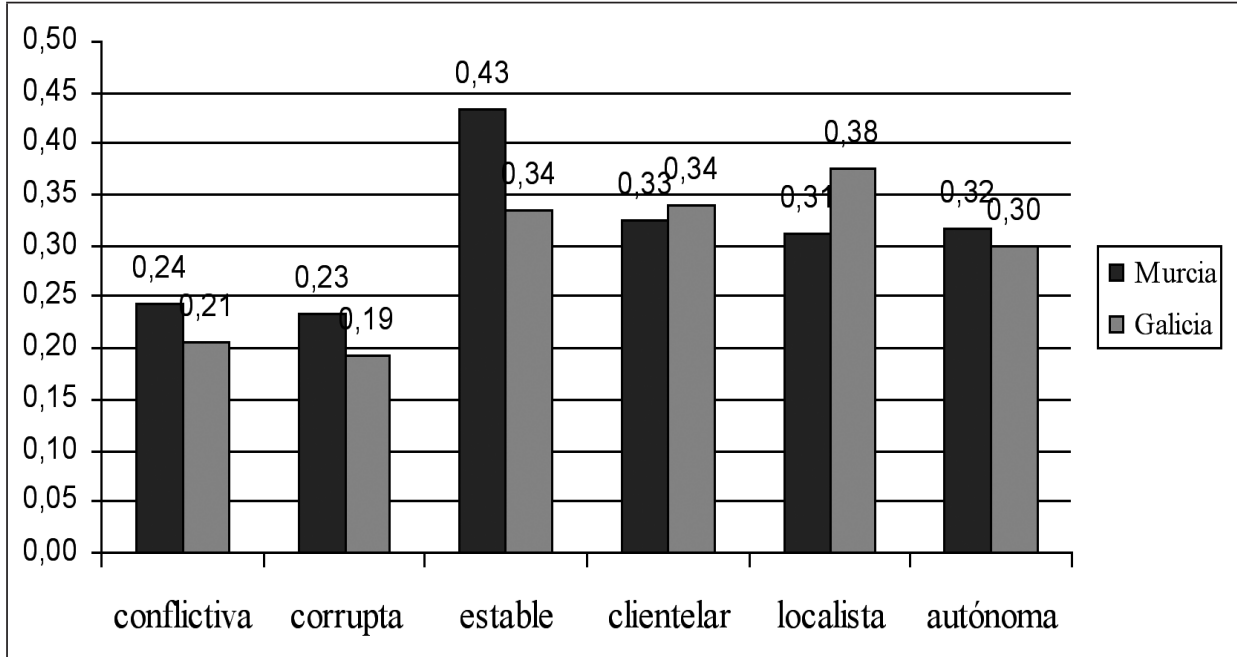

Fuente: Elaboración propia.

También preguntamos cómo definirían los entrevistados la vida política en la región, proponiéndoles seis características distintas a las que asignar un valor según fuera apropiado en una escala de 1 a 10: conflictiva, corrupta, estable, clientelista, local 
y autónoma. En ambas regiones ninguna de estas características obtuvo mucho apoyo entre los entrevistados (la puntuación más alta nunca llega a alcanzar el cinco). En ambas regiones, el clientelismo se encuentra entre las características de la política regional con mayor puntuación, aunque es un poco mayor en Galicia, lo que sugiere la persistencia de estructuras sociales y prácticas políticas tradicionales subyacentes al capital social heredado. Por otra parte, la percepción de la existencia de conflicto y corrupción es mayor en Murcia que en Galicia. Como mostraba el análisis de red, la participación más plural y activa de los actores sociales en Murcia podría explicar el mayor nivel de conflicto percibido por los entrevistados. También es importante subrayar la relevancia del conflicto del agua en la Región de Murcia, que se ha convertido en una cuestión clave del enfrentamiento político respecto al futuro de la región (surgiendo habitualmente en las entrevistas realizadas). En Galicia, en cambio, el conflicto se concentra básicamente en el sector laboral, de acuerdo con la clásica división entre asociaciones sindicales y empresariales. Asimismo, en Murcia -como en gran parte de la costa mediterránea- la corrupción está asociada básicamente con la construcción inmobiliaria y las políticas locales de urbanización.

Por último, los actores participantes de la política regional en Murcia y en Galicia comparten opiniones muy similares sobre la política regional de financiación europea. La percepción sobre la actividad realizada por el gobierno autonómico en relación con las políticas estructurales europeas es tan positiva en Murcia como en Galicia. De hecho, en ambas regiones casi el 70\% de los entrevistados evalúa positivamente las tareas llevadas a cabo por los gobiernos regionales. Es interesante advertir el mayor número de actores que en Galicia declaran no disponer de la suficiente información para evaluar cada uno de los PORs, lo cual parece reforzar la menor implicación de la participación efectiva de los actores de la sociedad civil en la elaboración de la política regional en esta región. Asimismo, mientras que los actores entrevistados en Galicia apenas expresan opiniones en contra del gobierno, aprobando mayoritariamente el trabajo realizado en los dos últimos programas operativos, en Murcia diversos actores juzgan negativamente cualquiera de los períodos financieros de los PORs. Valoraciones que hay que asociar con el mayor nivel de conflicto y de participación social que observamos en la red de política regional de Murcia en comparación con la gallega.

\section{CONCLUSIONES}

El ingreso en la Unión Europea y la consolidación de las comunidades autónomas han provocado transformaciones relevantes en la política regional en España. Actualmente, las regiones españolas gozan de una mayor autonomía en el diseño de sus estrategias de desarrollo que hace dos décadas. Sin embargo, su autonomía se encuentra limitada por dos aspectos básicos. Por un lado, la Constitución establece límites sobre la actividad del gobierno regional en la elaboración de la política económica; y por otro, al aumentar sus responsabilidades, las restricciones presupuestarias generan gobiernos

114 regionales más dependientes de las transferencias financieras nacionales y europeas. 
Un cambio que consideramos especialmente clave tiene que ver con la fase de programación de la política regional. La europeización del proceso de elaboración de políticas ha abierto la posibilidad de participación de los actores regionales en el diseño de la estrategia regional. Actualmente, los documentos estratégicos regionales están sujetos a procedimientos abiertos y participativos dentro de un proceso dirigido por el gobierno regional. Como hemos observado en los casos de Galicia y de la Región de Murcia, los gobiernos autonómicos han aprovechado esta oportunidad y se han convertido progresivamente en el actor principal, a pesar de que el nivel central todavía juega un papel de considerable importancia.

Hemos comprobado que los procesos formales de la política regional se repiten de igual manera en las dos comunidades estudiadas. Después de haber experimentado cuatro periodos financieros de la Política de Cohesión europea, los procedimientos se repiten y las tareas han sido bien asimiladas por las administraciones públicas autonómicas. No obstante, la red de actores de la política regional de ambas comunidades muestra una estructura bastante diferente: el índice de centralización de la red indica que la red que se configura en Galicia se encuentra mucho más centralizada que en Murcia. En particular, mientras que en Galicia las distintas unidades gubernamentales muestran una posición central altamente relevante en la red, en el caso de Murcia, por el contrario, las unidades gubernamentales no son tan centrales, con la única excepción de la Consejería de Economía. Las interacciones horizontales entre los actores sociales y las dinámicas de abajo hacia arriba son más frecuentes en Murcia que en Galicia, lo cual indica un patrón de elaboración de políticas más pluralista en el caso murciano.

Partiendo de estas caracteristicas diferenciales, podemos considerar que la estructura de la red en la politica regional de Galicia está dominada por la posición y las relaciones de los actores gubernamentales, que definen una policy community con una fuerte base estatista. Predomina un consenso básico entre los actores que integran la red, que articulan un patrón de relaciones intergubernamentales determinado en buena parte por las reglas formales de distribución del poder. Por el contrario, en el caso de la Región de Murcia se ha configurado una red donde la participación de actores no gubernamentales muestra un carácter más horizontal, con una estructura de relaciones más pluralista, lo que produce que la percepción de conflicto y competencia entre los actores sea más propia de una categoría de lobbying network. Una cierta diversidad de nucleos de influencia y capacidad de decisión fomenta la elevada intensidad de relaciones entre el conjunto de actores relevantes.

La mayor institucionalización que cabe atribuir al Gobierno autonómico de Galicia en comparación con el de la Región de Murcia, debido fundamentalmente a contar desde el inicio con el máximo techo competencial y, por tanto, con una maquinaria burócratica de importante volumen, puede explicar, en parte, el patrón de policy network muy próximo a las relaciones intergubernamentales que hallamos en Galicia. La menor institucionalización relativa del Gobierno murciano, y la consiguiente menor concentración del poder y de influencia en los actores gubernamentales, explica- 
ría el impulso de una red de política regional más plural, abierta y competitiva en esta comunidad autónoma.

Hemos observado que, de manera congruente con las características estructurales de cada red, las percepciones sobre la influencia que tienen los actores sociales varían también en las dos comunidades. Mientras la mayor parte de los actores entrevistados en la Región de Murcia opinan que las organizaciones sociales y los grupos de interés regionales ejercen influencia en la política regional, más de la mitad de los entrevistados en Galicia considera que las organizaciones sociales regionales apenas influyen en el proceso decisorio. Por otra parte, el análisis de los componentes de capital social de los actores participantes en la elaboración de la política regional aporta diferencias singificativas entre las dos comunidades autónomas, sugiriendo distintos patrones de interacción entre los actores. En Murcia los entrevistados muestran una mayor confianza en las instituciones públicas y también, aunque en menor medida, una mayor confianza social que en Galicia, al tiempo que también presentan mejores resultados en cuanto al capital social como "vínculo", es decir, el que favorece el intercambio y la cooperación en la esfera pública democrática. Puesto que partíamos de la observación de que, a principios de la década, el capital social de la población en general era muy similar en ambas regiones, cabe plantear la posibilidad de que la diferente configuración de la policy network de desarrollo regional en Galicia y en la Región de Murcia haya estimulado con éxito desigual los recursos de capital social entre las elites políticas y las organizaciones sociales de sendas comunidades.

En definitiva, si bien el proceso de europeización de las políticas de desarrollo regional ha representado la adopción de unas prácticas y procedimientos homogéneos respecto a la elaboración e implementación de la Política de Cohesión, las condiciones específicas de cada arena política regional han promovido una configuración específica de la policy network en la fase de programación de los Programas Operativos Regionales. La estructura y configuración de la policy network de desarrollo regional, de qué manera realiza el principio político de "partenariado", ha incidido en la toma de decisiones para la formulación de la política regional, y uno de los mecanismos que sin duda intervienen es el capital social de los actores implicados, un recurso crucial para la cooperación política y el rendimiento democrático.

\section{REFERENCIAS BIBLIOGRAFICAS Y DOCUMENTALES}

Alcaide, J. y Alcaide, P. (2003), Evolución Económica de las Comunidades Autónomas 1975-2000. Madrid: Instituto de Estudios Económicos.

Canova, F. (2001), “¿Contribuyen las políticas de la Unión Europea a estimular el crecimiento y a reducir las desigualdades regionales?”, Els Opuscles del CREI, 8, CREIUniversitat Pompeu Fabra.

Cienfuegos, M (2000), "La coordinación de los asuntos europeos en las administraciones autonómicas”, Revista de Estudios Politicos, 108: 103-142. 
Closa, C. y Heywood, P.M.(2004), Spain and the European Union. Londres: Palgrave Mac Millan.

Closa, C. (ed.) (2000), La europeización del sistema político español. Madrid: Istmo.

Comisión Europea (2003), Second Progress Report on Economic and Social Cohesion. Bruselas: Comisión Europea.

Comisión Europea (2004), A New Partnership for Cohesion. Convergence, Competitiveness, Cooperation. Third Report on Economic and Social Cohesión. Luxemburgo: Comisión Europea, Oficina de Publicaciones Oficiales de las Comunidades Europeas.

Comisión Europea (2005a), "Partnership in the 2000-2006 programming period”, Discussion paper of DG Regio. Bruselas: Comisión Europea.

Comisión Europea (2005b) Third Progress Report on economic and Social Cohesion. Bruselas: Comisión Europea.

Comisión Europea (2007) Fourth Progress Report on Economic and Social Cohesion. Bruselas: Comisión Europea.

Correa, M. D. y Manzanedo, J. (2002), Política regional española y europea. Periodo 1983-1999. Documento de Trabajo SGFCC-2002-05. Madrid: Dirección General de Presupuestos, Ministerio de Hacienda.

Cote S. y Healy, T. (2001), The Well-being of Nations. The role of human and social capital. París: OCDE.

Dastis, A. (1995), “La Administración española ante la Unión europea”, Revista de Estudios Políticos, 90: 323-349.

Dudek, C. M. (2005), EU Accession and Spanish Regional Development. Winners and Losers. Bruselas: Peter Lang S.A.

Eurostat (2008), Regional Statistics, <http://epp.eurostat.ec.europa.eu> (consulta: junio 2008).

Fernández-Fernández, J.(2004), “Las Intervenciones del Fondo de Desarrollo Regional (FEDER) en España durante el periodo de programación 2000-2006”, Noticias de la Unión Europea, 233: 9-36.

Fundación Galicia Europa (2007), Memoria 2006. Santiago de Compostela: FGE.

Heywood, P. y Molina I. (2000), “A quasi-presidential premiership: administering the executive summit in Spain”, en B.G. Peters, R.A.W. Rhodes y V. Wright, eds., Administering the Summit. Basingstoke: Palgrave. 
Herreros, F. (2004), The problem of forming social capital: Why trust? Nueva York: Palgrave.

Jordana, J. (1995), “El análisis de los policy networks: ¿Una nueva perspectiva sobre la relación entre políticas públicas y Estado?”, Gestión y Análisis de Políticas Públicas, 3: 77-90.

Leonardi, R. (2005), Cohesion policy in the European Union: the building of Europe, Londres: Palgrave.

Maravall, J.M. (1995), Los resultados de la democracia, Madrid: Alianza.

Marsh, D., ed. (1998), Comparing Policy Networks. Buckingham: Open University Press.

Marsh, D. y Rhodes, R.A.W. (eds.) (1992), Policy Networks in British Government. Nueva York: Oxford University Press.

Ministerio de Administraciones Publicas (2005), Las relaciones de colaboración EstadoComunidades Autónomas. Informe Anual 2004. Madrid: MAP.

Ministerio de Hacienda y Economía (2001), Marco Comunitario de Apoyo (20002006 para la Regiones Españolas del Objetivo 1). Madrid: Ministerio de EconomíaFEDER.

Ministerio de Hacienda y Economía (2005), La programación regional y sus instrumentos. Informe anual 2004. Madrid: MHE.

Molina, I. (1999), "The Impact of EU Membership on Domestic Policy-Making Process: Multi-level policy networks and central Coordination in Spain”, Paper for the Centre for European Studies 26-28, Universidad de Harvard.

Montero, J.R., Font, J. y Torca, M. (eds.) (2006). Ciudadanos, asociaciones y participación en España. Madrid: Centro de Investigaciones Sociológicas.

Morata, F (1998), La Unión Europea. Procesos, políticas, actores. Barcelona: Ariel.

Morata, F. y Muñoz, X. (1996), "Vying for European Funds: Territorial Restructuring in Spain”, en L. Hooghe, ed., Cohesion Policy and European Integration: Building Multi-Level Governance. Londres: Claredon Press.

Mota, F. y Subirats, J. (2000), “El quinto elemento. El capital social de las Comunidades Autónomas", Revista Española de Ciencia Política y de la Administración, 2: 123-158.

Mota, F. (2008), Capital social y gobernabilidad en el rendimiento político autonómico. $118 \quad$ Madrid: Centro de Estudios Políticos y Constitucionales. 
Narayan, D. (1999), Bonds and Bridges: Social capital and Poverty. Washington DC: World Bank.

OCDE (2005), OECD Economic Survey: Spain. Paris: OCDE.

Oppenheim, A. (1992), Questionnaire Design, Interviewing and Attitude Measurement, Londres: Pinter.

Piedrafita, S., Steinberg, F. y Torreblanca, J. I. (2007), 20 años de España en la Unión Europea (1986-2006). Madrid: Real Instituto Elcano.

Putnam, R. (2000), Bowling Alone: The Collapse and Revival of American Community. Nueva York: Simon \& Schuster.

Putnam, R., Leonardi, R. y Nanetti, R. (1993), Making Democracy Work. Civic Traditions in Modern Italy. Princeton: Princeton University Press.

Ramió C. y Salvador, M. (2002), “La configuración de las administraciones de las comunidades autónomas; entre la inercia y la innovación institucional”, en J. Subirats y R. Gallego, eds., Veinte años de autonomías en España. Madrid: CIS.

Región de Murcia (2004), Crecemos con Europa. Los Fondos Europeos en la Región de Murcia. Murcia: Dirección General de Presupuestos, Fondos Europeos y Finanzas, Consejeria de Hacienda.

Región de Murcia (2006), Plan estratégico de la Región de Murcia 2007-2013. <http:// www.horizonte2010.carm.es/neweb2/servlet/integra.servlets.ControlPublico?I DCONTENIDO=2799\&IDTIPO=100\&RASTRO=c\$m> (consulta: junio 2008).

Scharpf, F. (1999), Gobernar en Europa. ¿Eficaz y democráticamente? Madrid: Alianza.

Serrano Martínez, J.M. (2005), “Convergencia regional y polarización territorial en España. Un devenir complejo”, Boletín Económico del ICE 10/16 (2830): 17-32.

Sosvilla-Rivero, S. y Herce, J.A. (2004), “La política de cohesión europea y la economía española: evaluación y prospectiva”, Documento de Trabajo Real Instituto Elcano 142/204. Madrid: Real Instituto Elcano.

Subirats, J. y Gallego, R. (eds.) (2002), Veinte años de autonomías en España. Madrid: CIS.

Thielemann, E. (1998), “Policy networks and European governance: the europeanisation of regional policy-making in Germany", Regional and Industrial Research Paper Series, 27. Glasgow: European Policies Research Centre. 
Ward, S. y Williams, R (1997), “From Hierarchy to Networks? Sub-Central Government and EU Urban Environment Policy", Journal of Common Market Studies 35 (3):439464.

Wasserman, S. y Faust, C. (1998), Social Network Analysis. Methods and Applications. Cambridge: Cambridge University Press.

Woolcock, M. (2001), “The place of social capital in Understanding Social and Economic Outcomes”, Canadian Journal of Policy Research 2 (1): 11-17.

Xunta de Galicia (2000), Plan de Desenvolvementi Economico de Galicia 2000-2006. <http://www.xuntaeco.es/ga/pub/pedega/pedega.htm> (consulta junio 2008).

Zapico, E. (1995), “La adaptación de la administración publica española a la Unión Europea: un proceso de evolución y aprendizaje permanente”, Gestión y Análisis de Políticas Publicas 3: 43-54.

Zaragoza, J.A. (1990), “El resurgimiento de la política regional: la nueva política comunitaria y la política regional española”, Información Comercial Española, 679: 2737. 


\section{ANEXO}

\section{PRINCIPALES ACTORES}

\section{Región de Murcia}

\begin{tabular}{ll} 
DelegGob & Delegación del Gobierno Central en Murcia \\
ConsPres & Consejería de la Presidencia \\
OfRepM & Oficina de la Representación Regional en Bruselas \\
ConsEco & Consejería de Economía \\
ConsInd & Consejería de Industria y Medioambiente \\
ConsTrab & Consejería de Trabajo, Consumo y Política Social \\
INFO & Instituto de Fomento (dependiente de la Consejería de Industria y \\
FedMuni & Medioambiente) \\
AyuMur & Ayuntamientode Murcia \\
Camara & Cámara de Comercio de la Ciudad de Murcia \\
CC.OO & Sindicato \\
UGT & Sindicato \\
CROEM & Asociación de empresarios regional \\
InstMuj & Instituto de la Mujer \\
AMUSAL & Asociación de empresarios cooperativistas \\
UnivM & Universidad de Murcia \\
UnivPC & Universidad de Cartagena \\
Foro & Ong \\
Fund SM & Ong \\
\hline
\end{tabular}

\section{Región de Galicia}

ConsPres Consejería de la Presidencia

ConsEco Consejería de Economía

ConsIndu Consejería de Industria e Innovación

ConsTra Consejería de Trabajo

RelExts Secretaría de Acción Exterior (Consejería de la Presidencia) 
Jacint Jordana, Fabiola Mota y Andrea Noferini

\begin{tabular}{|c|c|}
\hline ConsEdu & Consejería de Educación \\
\hline IGAPE & $\begin{array}{l}\text { Instituo Gallego para la Promoción Económica/Agencia de Desarrollo } \\
\text { Regional }\end{array}$ \\
\hline Fund G-E & Fundación Galicia-Europa \\
\hline FEGAMP & Federación Gallega de Municipios y Provincias \\
\hline Eixo Atl & $\begin{array}{l}\text { Asociación Translimítrofe de las Municipalidades Portuguesas y Galle- } \\
\text { gas }\end{array}$ \\
\hline Zona Fra & $\begin{array}{l}\text { Consorcio Público/Agencia de Desarrollo Local (Zona de Libre Comer- } \\
\text { cio de Vigo) }\end{array}$ \\
\hline Camara & Cámara de Comercio de Santiago de Compostela \\
\hline DipACo & Diputación de A Coruña \\
\hline CES & Consejo Social y Económico de Galicia \\
\hline CCOO & Sindicato \\
\hline UGT & Sindicato \\
\hline $\mathrm{CIG}$ & Sindicato (regional) \\
\hline CEG & Asociación Empresaria Regional \\
\hline IGADI & Instituto Gallego de Análisis y Documentación Internacional \\
\hline USC & Universidad de Santiago de Compostela \\
\hline
\end{tabular}

